\title{
Water entry of a flat elastic plate at high horizontal speed
}

\author{
M. Reinhard $\dagger$, A. A. Korobkin and M. J. Cooker \\ School of Mathematics, University of East Anglia, Norwich NR4 7TJ, UK \\ (Received 30 June 2012; revised 8 March 2013; accepted 11 March 2013; \\ first published online 29 April 2013)
}

The two-dimensional problem of an elastic-plate impact onto an undisturbed surface of water of infinite depth is analysed. The plate is forced to move with a constant horizontal velocity component which is much larger than the vertical velocity component of penetration. The small angle of attack of the plate and its vertical velocity vary in time, and are determined as part of the solution, together with the elastic deflection of the plate and the hydrodynamic loads within the potential flow theory. The boundary conditions on the free surface and on the wetted part of the plate are linearized and imposed on the initial equilibrium position of the liquid surface. The wetted part of the plate depends on the plate motion and its elastic deflection. To determine the length of the wetted part we assume that the spray jet in front of the advancing plate is negligible. A smooth separation of the free-surface flow from the trailing edge is imposed. The wake behind the moving body is included in the model. The plate deflection is governed by Euler's beam equation, subject to free-free boundary conditions. Four different regimes of plate motion are distinguished depending on the impact conditions: $(a)$ the plate becomes fully wetted; $(b)$ the leading edge of the plate touches the water surface and traps an air cavity; $(c)$ the free surface at the forward contact point starts to separate from the plate; $(d)$ the plate exits the water. We could not detect any impact conditions which lead to steady planing of the free plate after the impact. It is shown that a large part of the total energy in the fluid-plate interaction leaves the main bulk of the liquid with the spray jet. It is demonstrated that the flexibility of the plate may increase the hydrodynamic loads acting on it. The impact loads can cause large bending stresses, which may exceed the yield stress of the plate material. The elastic vibrations of the plate are shown to have a significant effect on the fluid flow in the wake.

Key words: flow-structure interactions, high-speed flow, wakes/jets

\section{Introduction}

We analyse the interactions that occur when an elastic plate impacts the free surface of deep water (see figure 1). We are especially interested in investigating the interactions between the plate and the fluid when the plate has a small angle of attack and a forward speed that is much larger than its velocity component of descent onto the water. In this class of impacts the hydrodynamic loads are very high and 


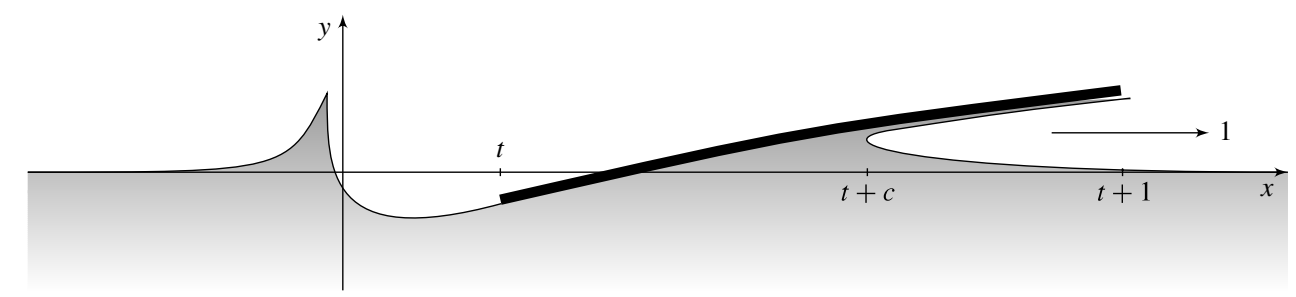

FIGURE 1. Plate impact onto deep water at time $t>0$ after the initial touch-down of the trailing edge. Note the splash region close to the origin, the trailing edge, the jet thrown forwards from the turnover point $x=t+c$ and the front edge. The plate freely descends and has unit horizontal velocity component.

the flexibility of the plate may play a significant role in the interaction. Such impacts can be observed in the slamming of high-speed vessels. Ulstein (1995) investigated the fluid interaction with a flexible stern seal bag on surface-effect ships, which alternately jump out of and re-enter the water (cobblestone effect). The aircraft industry is interested in the hydrodynamic processes in both regular landing of seaplanes and for the safe landing of aircraft onto water during emergencies. The slamming loads in all these problems may cause unwanted structural responses. The elasticity of the body may significantly influence the interaction between fluid and body.

\subsection{Experimental studies}

In contrast to many experiments on vertical impact and planing, we know of only a few reports on plate impact experiments at high horizontal velocity. The impact experiments by Smiley (1951) with a rectangular plate landing on water at high horizontal speed showed that the hydrodynamic pressure acting on the plate is close to uniform in the transverse direction. The measured pressure drops to the ambient value just near the transverse edges of the plate. This supports our modelling in two dimensions. Rosselini et al. (2005) conducted experiments on skipping stones. They showed, for a stone without spin, that its angle of attack can decrease to negative values during the impact so that the stone can dive into the water. The behaviour of a paddle on the surface of a shallow stream has been investigated experimentally and compared with an analytic model in Hewitt, Balmforth \& McElwaine (2011). Their experiments showed that the paddle oscillates for large enough stream velocities and can take off into a continual bouncing motion.

\subsection{Oblique water impact and unsteady planing}

The oblique impact of a semi-infinite rigid plate with constant velocity onto a flat free surface is self-similar. Faltinsen \& Semenov (2008) presented the method and numerical results for a wide range of angles of attack of such a plate. Sedov (1940) and Ulstein \& Faltinsen (1996) studied oblique impacts of a rigid plate for small angles of attack with prescribed vertical motion. Their results are reproduced with our model for constant vertical velocity of the plate. Oblique impacts of wedges and parabolas with small deadrise angle into deep water with constant speed have been considered by Oliver (2002), Howison, Ockendon \& Oliver (2004) and Moore et al. (2012a) in the initial stage when jets appear at the front and the rear of the body. Three-dimensional impact problems with horizontal speed, mainly with cones 
and elliptic paraboloids, have been discussed in Moore et al. (2012b) and Scolan \& Korobkin (2012).

In a shallow-water approximation, Hicks \& Smith (2011) investigated the free impact of a rigid plate at high horizontal speed. In particular, they showed that the plate can exit the fluid unless the plate gets fully wetted. This is in agreement with our deep-water analysis, where basically the same flow regimes are observed. The impact of an ellipse into shallow water at high horizontal speed has been investigated in Khabakhpasheva \& Korobkin (2013).

\subsection{Wagner model of water impact and spray jet}

The water impact under investigation is characterized by a turnover region of the free-surface flow, where a thin jet is formed adjacent to the plate and thrown forward of the advancing plate (see figure 1). The thickness of the spray jet and the size of the turnover region are proportional to $\alpha^{2}$ for a small angle of plate inclination $\alpha$ (Howison, Ockendon \& Wilson 1991), so that at the turnover point the free-surface elevation is approximately equal to the elevation of the plate. This fact was exploited by Wagner (1932) to determine the position of the turnover region within the linearized hydrodynamic problem. The high-speed jet gives only a small contribution to the loads acting on the plate and we neglect it. For a wedge entering water at constant vertical speed, Zhao \& Faltinsen (1993) showed that the pressure distributions obtained from Wagner's approach agree well with the nonlinear self-similar solution for small deadrise angles. By analysing the local flow in the turnover region, Wagner (1932) corrected the square-root singularity of the pressure at the contact points predicted within his linearized model. Several modifications of the original Wagner theory (e.g. Cooker 1996; Vorus 1996; Zhao, Faltinsen \& Aarsnes 1996; Korobkin 2004) were introduced to improve the theoretical predictions of the hydrodynamic impact loads acting on different body shapes entering water. The original Wagner model solves the impact problem in the leading order of the small deadrise angle. Second-order extensions have been presented by Korobkin (2007) and Oliver (2007). They found that the second-order corrections of the force lead to significant improvements of the predictions. They showed that the second-order terms for the positions of the turnover region are small and zero in the impact of a wedge and parabola, respectively.

\subsection{Separation of the free surface at the trailing edge of the plate}

In oblique plate impact a splash appears behind the trailing edge at the point where the plate first touches the free surface. The part of the free surface between the initial splash and the advancing trailing edge of the plate (see figure 1) is referred to as the wake. Experiments confirm a smooth flow separation from a sharp trailing edge for planing plates at high horizontal velocity, corresponding to Kutta's condition of finite flow speed at a sharp edge (see e.g. Newman 1977, p. 171).

When the hydrodynamic pressure along the plate is much less than atmospheric pressure, then the fluid may separate from the plate in the low-pressure zone. This phenomenon may lead to cavitation if a low-pressure zone is well within the contact region, distant from the trailing edge, or ventilation may occur if the low-pressure zone is attached to the trailing edge. Both cavitation and ventilation are important issues for high-speed vessels, as discussed in Faltinsen (2005). Ventilation in oblique impact problems has been accounted for in Semenov \& Yoon (2009) and Reinhard, Korobkin \& Cooker $(2012 b)$. In the present model, the fluid is assumed to be attached to the 
plate from the turnover region to the trailing edge, so that any suction forces of the fluid contribute to the dynamics of the plate.

\subsection{Elastic-plate interaction with water during impact}

It is common to model the flexibility of a thin plate by Euler's beam equation (see Donnell 1976). The first models of an elastic structure coupled with impact onto a liquid free surface are by Meyerhoff $(1965 a, b)$. In these papers the vertical impact of an elastic symmetric wedge was addressed using a normal-mode expansion of the body deflection. A similar approach was used in the problem of wave impact onto an elastic plate, as studied by Faltinsen, Kvålsvold \& Aarsnes (1997), Korobkin (1998) and Korobkin \& Khabakhpasheva (2006). Owing to the elastic deformation of the plate, the contact-point motion and the loads acting on the plate can differ significantly from those for a rigid plate. Interactions of a flexible-body stern seal bag with the water free surface at high horizontal speed were analysed by Ulstein (1995). However, to couple the structural part with the hydrodynamic part, he approximated the vertical bag motion. In the present paper, we overcome the difficulties in the coupling by finding an explicit form for the hydrodynamic pressure under the plate. To show certain hydroelastic features we will determine simultaneously the flow generated by the impact, the hydrodynamic loads acting on the plate, the size of the contact region between the plate and the fluid, the rigid-body motion, and the elastic deflections of the plate.

\subsection{Global characteristics of elastic-plate impact with horizontal speed}

It is well known that the energy conservation law is not satisfied within the classical Wagner theory when the kinetic energy of the jet is not included (see Scolan \& Korobkin 2003). The jet energy is not small and can be accounted for with the help of a local analysis of the flow in the turnover region. In the problem of the vertical water entry of a sphere at constant speed, Cointe et al. (2004) showed that half of the work done by the sphere on the fluid is expressed in the spray jet and the other half is the kinetic energy of the bulk of the flow domain. The corresponding two-dimensional problem of a parabolic body entering water at constant speed was investigated by Korobkin (1994), who arrived at the same equipartition of energy. We will show that this equipartition is not satisfied, if the body moves with non-zero horizontal velocity component.

For large horizontal speed of a free plate impacting a shallow liquid layer with small angle of attack, Hicks \& Smith (2011) showed that this plate moves with a constant horizontal velocity to leading order. However, even for a small decrease of the horizontal speed, the loss of kinetic energy due to horizontal deceleration of the plate might be of the same order as other components of the plate and liquid energies. In the present analysis, the plate moves with constant horizontal velocity component, $U$. Therefore, a horizontal force has to be applied to the centre of the plate to oppose the corresponding hydrodynamic force. The work done by this external force will be included in the energy equation.

An analysis of the energy components in the problem of wave impact onto an elastic plate which is slightly elevated above the still water surface, was presented by Korobkin \& Khabakhpasheva (2006). They concluded that the larger the potential energy of the deformed beam, the smaller is the kinetic energy in the spray jet. In the present problem, the decomposition of the energy and time evolutions of energy components are shown for two example cases. 


\subsection{Air-cushion effects and the flow generated by impact}

Air-cushioning effects have been considered in Hicks \& Purvis (2010) for droplet impact onto a wall at high tangential speed. They showed an unsymmetrical droplet deformation not allowing air to be trapped between the fluid and the wall if the speed of the droplet is large enough. However, in the present impact problem, we cannot exclude that air pockets may develop in the initial stage of impact. The present model does not account for the presence of air.

We only consider the impact stage, where the plate strongly interacts with the fluid, with the plate rigid and elastic motion being dependent on the hydrodynamic loads and these loads, in turn, being dependent on the plate motions, both rigid and elastic. The impact stage is defined as the early stage during which the plate is only partially wetted and the wetted portion of the plate grows at a high rate. For a plate of length $L$, inclined at a small angle $\alpha$ to the free surface and penetrating the liquid at vertical speed $V$, the duration of the impact stage can be estimated as $T=L \sin \alpha / V$. The vertical displacement of the plate during this stage is of the order of $L \sin \alpha$, which is much smaller than the length scale of the problem, $L$. This implies that the deformations of the liquid boundary are of the order of $L \sin \alpha$ and can be neglected in the leading order compared with $L$. The plate displacement along the free surface during the impact stage is of the order of $U L \sin \alpha / V=U T$. In the general case this displacement is of the order of the length scale $L$ of the problem, which gives $V / U=O(\alpha)$ for small inclination angle $\alpha$. Hence, the duration of the impact stage can be also estimated as $T=O(L / U)$ which is valid for $V / U \ll 1$.

For impact conditions with the plate length of order $1 \mathrm{~m}$, vertical velocity of order of $1 \mathrm{~m} \mathrm{~s}^{-1}$ and horizontal velocity of order $10 \mathrm{~m} \mathrm{~s}^{-1}$ the liquid can be modelled as inviscid and incompressible, and the flow initiated by the plate impact as irrotational and two-dimensional. We find that the Reynolds number is of order $10^{7}$ and the duration of the impact stage, $T$, is of order $0.1 \mathrm{~s}$. The impact stage is so short that any vorticity in the viscous boundary layer on the plate has no time to advect into the main part of the fluid domain. The ratio of the viscous time scale to $T$ is of order $R e^{-1}$. The viscous skin friction force is accordingly small; it is only $R e^{-1 / 2}$ times the horizontal component of the inertial force. In the impact stage, gravity has no time to influence either the velocity field or the pressure distribution in the fluid domain.

The splash shown in figure 1 on the left of the impact point $x=0, y=0$ was studied numerically by Faltinsen \& Semenov (2008). This splash is formed at the beginning of the impact, when the plate flexibility does not matter and the flow is self-similar with respect to $x / V t$ and $y / V t$. In the present study, we assume that the fine details of the flow in the splash do not affect the loads acting on the plate in the leading order.

\subsection{Overview of the contents}

The structure of the paper is as follows. We start with the mathematical formulation of the problem in $\S 2$. The plate motion is described by Euler's beam equation with free-free boundary conditions. The two-dimensional flow generated by the plate impact is governed by a mixed boundary-value problem for the velocity potential. We impose Wagner's condition at the turnover region, Kutta's condition and the condition of continuous free-surface separation at the trailing edge. The Euler beam equation is coupled with the hydrodynamic pressure which depends on the plate motion. The solution of the formulated coupled problem is outlined in $\S 3$. Three integral equations are derived by using the three conditions at the two ends of the contact region. The plate deflection is expressed in terms of the normal modes of elastic-beam vibration 
in a vacuum. The motion of the body is formulated with respect to the principal coordinates of the elastic beam deflection and two functions describing the rigid-body motion of the plate. The final system of the hydroelastic problem is outlined in $\S 4$. Section 5 explains how to apply the present theory to the problem when the plate is rigid and its velocity constant. In $\S 6$, by including the energy of the spray jet we confirm analytically the balance of the work applied to move the elastic plate at constant horizontal speed and the energy of the fluid-plate system. In $\S 7$ the different scenarios of the plate motion are analysed and the numerical results are discussed for two specific configurations of the plate. Conclusions are drawn and future work is suggested in $\S 8$.

\section{Mathematical formulation}

Initially the liquid is at rest and occupies the lower half-plane $y^{\prime}<0$. The elastic plate is initially undeformed, inclined to the liquid free surface at a small angle $\alpha$, and touches the free surface at a single point which is taken as the origin of the Cartesian coordinate system $x^{\prime} O y^{\prime}$. The global coordinates are fixed with respect to the far-field fluid, which is at rest. At time $t=0$ the plate starts to penetrate the liquid vertically at the initial velocity $V$, and to move horizontally with a constant sustained horizontal velocity component $U$, where $V / U=O(\alpha)$. The initial angular velocity is zero. The plate material has density $\varrho_{S}$, Young's modulus $E$ and Poisson's ratio $v$. The plate is of length $L$ and small thickness $h$, such that $h / L \ll 1$. The liquid is incompressible, inviscid and of constant density $\varrho_{F}$. The flow generated by the penetrating plate is assumed two-dimensional and potential. Gravity and surface tension are neglected in the hydrodynamic model. Gravity can be neglected in the leading-order model when the Froude number $U / \sqrt{g L}$ is large, where $g$ is the acceleration due to gravity. The presence of air is neglected. The atmospheric pressure is set to zero. We are concerned with the initial stage of the plate impact, whose duration is of order $L / U$. The ratio $L / U$ is taken as the time scale of the problem. The plate length $L$ is the length scale of the problem. The plate deflection is of order $\alpha L$. The fluid velocity is of order $\alpha U$, so that we scale the fluid velocity potential by $\alpha U L$. Pressure is scaled by $\alpha \varrho_{F} U^{2}$. Dimensional variables are denoted by primes and we drop the primes for the corresponding non-dimensional variables. The initial angle $\alpha$ of the plate inclination plays the role of a small parameter.

\subsection{Structural part of the problem}

The position of the plate at time $t$ is described, in the non-dimensional variables, by the equation

$$
y=\alpha \omega(x, t),
$$

which includes both the rigid-body motion of the plate and its elastic deflection. The function $\omega(x, t)$ is unknown in advance and must be determined together with the liquid flow and the hydrodynamic pressure. In the coordinate system of the plate, the shape of the plate is described by the equations

$$
y=\alpha \zeta(s, t), \quad s=x-t \quad(0 \leqslant s \leqslant 1)
$$

such that $\zeta(s, t)=\omega(s+t, t)$. We model the plate motions, both rigid and elastic, with Euler's beam equation (Donnell 1976)

$$
\mu \zeta_{t t}+\theta \zeta^{(\mathrm{iv})}=q(s, t) \quad(0<s<1)
$$


where $\mu=\varrho_{S} h \varrho_{F}^{-1} L^{-1}, \theta=D \varrho_{F}^{-1} L^{-3} U^{-2}$ and $D=E h^{3} /\left(12\left(1-v^{2}\right)\right)$ is the flexural rigidity of the plate. In (2.3), $\zeta^{\text {(iv) }}=\partial^{4} \zeta / \partial s^{4}$ and $q(s, t)$ is the external vertical load acting on the plate. Equation (2.3) is written in the moving coordinate system with the longitudinal coordinate along the plate being approximated as $x-t$, which is correct in the leading order for small inclinations of the plate. In the global coordinate system $x O y$, the moving plate corresponds to the interval $y=0, t<x<t+1$.

Only the bending stresses and the normal shear forces are taken into consideration in Euler's beam model. The ends of the plate are free of stresses and external forces, so we have the free-free boundary conditions (Donnell 1976)

$$
\zeta^{\prime \prime}=\zeta^{\prime \prime \prime}=0 \quad(s=0 \text { and } s=1)
$$

for (2.3). The primes in (2.4) are defined as the $s$-derivatives of $\zeta(s, t)$. The initial conditions for (2.3) and (2.4) are

$$
\zeta(s, 0)=s, \quad \zeta_{t}(s, 0)=-\chi,
$$

where $\chi=V /(\alpha U)$ is the non-dimensional initial vertical velocity of the plate. Tension in the plate is neglected, since the horizontal forces in the plate are much smaller than the vertical ones for the free-free plate. The problem (2.3)-(2.5) can be readily solved if the external vertical load $q(s, t)$ in $(2.3)$ is known. However, this is not the case. The loads on the plate resulting from the fluid flow should be determined together with the plate motion. The rigid-body motion of the plate and its elastic deflection will be evaluated in $\S 3.3$ by the normal-mode method.

\subsection{Hydrodynamic part of the problem}

A schematic of the flow produced by the plate impact is shown in figure 1 . The plate moves from left to right and penetrates the liquid. During the early stage of the interaction the deformation of the liquid boundary is small compared with the length $L$ of the body. This fact makes it possible to linearize the boundary conditions and impose them on the original position of the liquid boundary, $y=0$, in the leading order for small $\alpha$ (see Korobkin \& Khabakhpasheva 2006, for more details of this approximation). The velocity field is described by the velocity potential $\varphi(x, y, t)$. The hydrodynamic pressure $p(x, y, t)$ is given by Bernoulli's equation, which can be linearized during the initial stage of impact and in non-dimensional variables is

$$
p(x, y, t)=-\varphi_{t}(x, y, t) \quad(y \leqslant 0) .
$$

The hydrostatic term is neglected in (2.6) because of the small penetration depth of the plate. Within this model, which is referred to as the Wagner model of water impact (Wagner 1932), the thin jet formed in the overturning region (see figure 1) is not taken into consideration. Parameters of this jet can be determined after the flow in the main flow region has been obtained (Howison et al. 1991). The spatial interval $t \leqslant x \leqslant t+c(t)$ on $y=0$ corresponds to the wetted part of the moving plate. Note that the fluid also wets the plate outside this region in the nonlinear hydrodynamic model. Other names for the interval $t \leqslant x \leqslant t+c, y=0$ such as 'high pressure region on the plate', 'contact region', 'Wagner region', 'wetted area' and 'contact set' can be found in the literature. The point $x=t, y=0$ is the trailing edge of the plate, and the point $x=t+c(t), y=0$ is the overturning region referred to below as the Wagner contact point, also known as the 'free point' in codimension-two problems (Howison, Morgan $\&$ Ockendon 1997). Here the function $c(t)$ is unknown in advance and is determined as part of the solution. The speed $1+\dot{c}(t)$ is assumed positive during the early stage. The free-surface shape is described by the equation $y=\alpha \eta(x, t)$. 
The velocity potential $\varphi(x, y, t)$ satisfies the following equations which can be compared with the models by Ulstein \& Faltinsen (1996) and Howison et al. (2004) formulated in the frame of reference of the body:

$$
\begin{aligned}
\nabla^{2} \varphi & =0 \quad(y<0), \\
\varphi_{y} & =\omega_{t}(x, t) \quad(y=0, t<x<t+c), \\
\varphi & =0 \quad(y=0, x<0 \text { and } x>t+c), \\
\varphi & =\bar{\varphi}(x) \quad(y=0,0<x<t), \\
\varphi & \rightarrow 0 \quad\left(x^{2}+y^{2} \rightarrow \infty\right) .
\end{aligned}
$$

Equations (2.9) and (2.10) follow from the dynamic boundary condition $p=0$ on the free surface. Integrating this condition in time, and using the linearized Bernoulli equation and the initial conditions, we arrive at (2.9) and (2.10), where the wake function $\bar{\varphi}(x)$ must be determined as part of the solution. Equation (2.8) is the linearized body boundary condition.

We seek a velocity potential $\varphi(x, y, t)$ which satisfies $(2.7)-(2.11)$ and that has no singularities in the flow region $y \leqslant 0$. In the linearized hydrodynamic model, the fluid velocity and the hydrodynamic pressure are square-root singular at the Wagner contact point, $y=0, x=t+c(t)$. Such a solution of the problem (2.7)-(2.11) is uniquely defined for $t>0$, if the functions $c(t), \omega(x, t)$ and $\bar{\varphi}(x)$ are prescribed. However, this is not the case here. The shape of the elastic plate $\omega(x, t)$ is determined by (2.3)-(2.5). The external vertical load on the plate in (2.3) consists of the hydrodynamic pressure on the wetted part of the plate and the gravity force. In the leading order of small $\alpha$ we obtain

$$
q(s, t)= \begin{cases}p(s+t, 0, t)-\mu \kappa & \text { for } 0 \leqslant s<c, \\ -\mu \kappa & \text { for } c \leqslant s \leqslant 1,\end{cases}
$$

where $\kappa=g L \alpha^{-1} U^{-2}$ and $p(x, 0, t)$ is given by (2.6). This implies that the problem is coupled: the flow, the pressure distribution, the motion of the plate and its elastic deflection have to be determined simultaneously with the size of the contact region and the shape of the free surface (Korobkin \& Khabakhpasheva 2006).

The length of the wetted part of the plate, $c(t)$, is determined by using Wagner's condition (Wagner 1932) at $x=t+c$ :

$$
\eta(t+c, t)=\omega(t+c, t)
$$

where the free-surface elevation $\eta(x, t)$ is obtained from the time integral of the vertical velocity component in the free-surface kinematic boundary condition:

$$
\eta_{t}(x, t)=\varphi_{y}(x, 0, t) \quad(x<t, x>t+c)
$$

away from the moving plate. The wake function $\bar{\varphi}(x)$ in (2.10) is obtained with the help of Kutta's condition at the trailing edge

$$
|\nabla \varphi(t, 0, t)|<+\infty .
$$

It will be shown later that $\bar{\varphi}(0) \neq 0$ so that (2.9) implies a jump of $\varphi(x, 0, t)$ at $x=0$. It follows from (2.7)-(2.11) that the vertical velocity component of the fluid is logarithmic singular at the origin. To resolve this singularity, an inner region at $x=0, y=0$ is required. However, we assume that details of the local flow in this inner region do not affect the hydrodynamic loads acting on the plate. It is these hydrodynamic loads which are of major concern in this study. Furthermore, we impose 
the condition of continuous separation of the free surface from the trailing edge, at $x=t, t>0$ :

$$
\eta(t, t)=\omega(t, t) .
$$

Condition (2.16) is needed to integrate (2.14) in the wake region, $0<x<t$. Note the similarity of the conditions (2.13) and (2.16) imposed at the end points of the contact region.

The solution of the coupled problem (2.3)-(2.16) depends on four parameters: $\chi, \mu$, $\theta$ and $\kappa$. The limit $\theta \rightarrow \infty$ corresponds to the entry of a rigid plate. For $\mu \rightarrow \infty$ the structural mass of the plate $\varrho_{S} h L$ is much greater than the corresponding added mass $\varrho_{F} L^{2}$, so that the hydrodynamic pressure does not influence the motion of the plate. In particular, the case $\mu \rightarrow \infty$ and $\kappa=0$ corresponds to the problem where a rigid plate impacts with constant velocity. This problem will be separately discussed in $\S 5$. If $\mu$ and $\theta$ are small, then this corresponds to a light flexible plate and the problem should be solved by methods of asymptotic analysis, which we do not pursue in this paper. Our analysis will treat both rigid and elastic motions of the plate, and the associated hydrodynamic loads. A direct numerical solution is difficult because the problem is nonlinear, coupled and unknown functions are singular.

\section{Solution of the coupled problem}

The problem consists of three parts: (a) hydrodynamic part, equations (2.6)-(2.11) and (2.15); (b) a kinematic part which provides a solution of Wagner's condition (2.13), (2.14) and identifies the unique solution of the coupled problem, which satisfies condition (2.16); (c) a structural part, (2.3)-(2.5) and (2.12), describing the plate deflection caused by the hydrodynamic load. The solutions of these three problems are outlined below and linked together at the end of the section.

\subsection{Hydrodynamic problem}

Equations (2.7)-(2.11) are a mixed boundary-value problem for the complex velocity $\varphi_{x}-\mathrm{i} \varphi_{y}$. This function is analytic in $y<0$ and decays to zero in the far field. The solution of this problem can be obtained by using the theory of boundary problems for analytic functions (see Gakhov 1966; King 2009). In particular, we obtain that the horizontal component of the liquid velocity in the contact region, $y=0, t<x<t+c(t)$, between the fluid and the plate is given by

$$
\varphi_{x}(x, 0, t)=\frac{G(x, t)}{\pi \sqrt{(x-t)(t+c-x)}}
$$

where

$$
\begin{aligned}
G(x, t)= & \int_{0}^{t} \frac{\sqrt{(t-\xi)(t+c-\xi)}}{\xi-x} \bar{\varphi}_{x}(\xi) \mathrm{d} \xi \\
& +f_{t}^{t+c} \frac{\sqrt{(\xi-t)(t+c-\xi)}}{\xi-x} \omega_{t}(\xi, t) \mathrm{d} \xi .
\end{aligned}
$$

Here, a dash in the integral sign denotes a Cauchy principal-value integral (Gakhov 1966). Eigensolutions of the problem (2.7)-(2.11) with $\omega(x, t) \equiv 0$ and $\bar{\varphi} \equiv 0$ (see Iafrati \& Korobkin 2008) are not included in (3.1) because they have stronger singularities at the end points $x=t$ and $x=t+c$ than in (3.1), which are not permitted by conditions (2.13) and (2.16). 
Kutta's condition (2.15) and (3.1) yield $G(t, t)=0$. Then (3.2) at $x=t$ gives a singular Volterra equation of the first kind with respect to the horizontal velocity $\bar{\varphi}_{x}(x)$ in the wake, $0<x<t$ :

$$
\int_{0}^{t} \sqrt{\frac{t+c-\xi}{t-\xi}} \bar{\varphi}_{x}(\xi) \mathrm{d} \xi=\int_{t}^{t+c} \sqrt{\frac{t+c-\xi}{\xi-t}} \omega_{t}(\xi, t) \mathrm{d} \xi .
$$

This equation will be used later to determine the wake function $\bar{\varphi}_{x}(x)$. The solution of the hydrodynamic problem (2.7)-(2.11) subject to Kutta's condition (3.3) shows that $\varphi_{x}(x, 0, t)$ is continuous at $x=t$. It will be shown later that the fluid is moving from the trailing edge to the wake with high velocity in the body's frame of reference. The wake velocity $\bar{\varphi}_{x}(t)$ at the trailing edge depends on the vertical velocity of the plate $\omega_{t}(x, t)$ which is unknown in advance, and the length $c(t)$ of the contact region, as shown in the integral equation (3.3).

By integrating (3.1) with respect to $x$ with the condition $\varphi(t+c, 0, t)=0$ and using (2.6) and (3.3), we obtain the pressure distribution along the wetted part of the plate as

$$
p(x, 0, t)=-\frac{1+\dot{c}}{\pi c} \sqrt{\frac{x-t}{t+c-x}} B(t)-\frac{1}{\pi} \sqrt{(x-t)(t+c-x)} T(x, t),
$$

where

$$
\begin{aligned}
B(t) & =-G(t+c, t), \\
T(x, t) & =\int_{t}^{t+c} \frac{\hat{\omega}_{t t}(\xi, t)}{(\xi-x) \sqrt{(\xi-t)(t+c-\xi)}} \mathrm{d} \xi, \\
\hat{\omega}_{t t}(x, t) & =\int_{t}^{x} \omega_{t t}(\xi, t) \mathrm{d} \xi .
\end{aligned}
$$

We consider only the cases where $G(t+c, t)>0$ in (3.1). Then $B(t)$ in (3.5) is negative and the hydrodynamic pressure (3.4) is positive and close to square-root singular at the leading edge of the wetted region, $x=t+c$. Note that for large positive deflections of the elastic plate the function $G(t+c, t)$ may drop down to zero and even become negative (see (3.2)). If so, the leading edge $x=t+c$ should be treated as a point of the free-surface separation from the plate.

\subsection{Kinematic part of the problem}

To employ Wagner's condition (2.13) and the condition of continuous free-surface separation (2.16), we need to know the free-surface elevation $\eta(x, t)$. We could evaluate $\eta(x, t)$ by using the solution of the hydrodynamic problem from $\S 3.1$ and integrating with respect to $t$ the kinematic boundary condition (2.14). However, it is convenient to evaluate the kinematics of the free surface directly by introducing the displacement potential

$$
\Phi(x, y, t)=\int_{0}^{t} \varphi(x, y, \tau) \mathrm{d} \tau \quad(y \leqslant 0)
$$

and reformulating the hydrodynamic problem (2.7)-(2.11) with respect to this potential (see Howison et al. 1991). Then the free-surface elevation is given by

$$
\eta(x, t)=\Phi_{y}(x, 0, t),
$$


where $x<0$ or $x>t+c$. Integrating (2.7)-(2.11) in time, using the initial conditions and Wagner's condition (2.13), we derive the following mixed boundary-value problem:

$$
\begin{aligned}
\nabla^{2} \Phi(x, y, t) & =0 \quad(y<0), \\
\Phi_{y}(x, 0, t) & =\omega(x, t) \quad(t<x<t+c), \\
\Phi(x, 0, t) & =0 \quad(x<0, x>t+c), \\
\Phi_{x}(x, 0, t) & =(t-x) \bar{\varphi}_{x}(x)+A(x) \quad(0<x<t), \\
\Phi(x, y, t) & \rightarrow 0 \quad\left(x^{2}+y^{2} \rightarrow \infty\right),
\end{aligned}
$$

where $A(x)$ is an undetermined term due to the time-integration. The condition of continuous separation (2.16) together with (2.14) and (3.11) gives us the freesurface elevation (3.9) for $0<x<t$. We seek the solution $\Phi(x, y, t)$ of the problem (3.10)-(3.14) which is bounded together with its first derivatives $\Phi_{x}$ and $\Phi_{y}$ at the points $x=t, y=0$ and $x=t+c, y=0$. The derivatives $\Phi_{x}(x, y, t)$ and $\Phi_{y}(x, y, t)$ provide the components of displacement of a liquid particle.

The boundary-value problem (3.10)-(3.14) for $\Phi(x, y, t)$ is similar to the problem (2.7)-(2.11) for $\varphi(x, y, t)$. By using the same techniques, we find the elevation of the free surface in the form

$$
\eta(x, t)= \pm \frac{E(x, t)}{\pi \sqrt{(x-t)(x-t-c)}}
$$

where

$$
\begin{aligned}
E(x, t)= & \int_{0}^{t} \frac{\sqrt{(t-\xi)(t+c-\xi)}}{\xi-x} \Phi_{x}(\xi, 0, t) \mathrm{d} \xi \\
& +\int_{t}^{t+c} \frac{\sqrt{(\xi-t)(t+c-\xi)}}{\xi-x} \omega(\xi, t) \mathrm{d} \xi .
\end{aligned}
$$

The plus sign in (3.15) applies for $x>t+c$ and the minus sign for $x<t$. Conditions (2.13) and (2.16) show that $E(t+c, t)=0$ and $E(t, t)=0$. The formula (3.16) applied at $x=t+c$ and $x=t$ yields two integral equations

$$
\begin{aligned}
& \int_{0}^{t} \sqrt{\frac{t-\xi}{t+c-\xi}} \Phi_{x}(\xi, 0, t) \mathrm{d} \xi=-\int_{t}^{t+c} \sqrt{\frac{\xi-t}{t+c-\xi}} \omega(\xi, t) \mathrm{d} \xi \\
& \int_{0}^{t} \sqrt{\frac{t+c-\xi}{t-\xi}} \Phi_{x}(\xi, 0, t) \mathrm{d} \xi=\int_{t}^{t+c} \sqrt{\frac{t+c-\xi}{\xi-t}} \omega(\xi, t) \mathrm{d} \xi
\end{aligned}
$$

with respect to the functions $A(x)$ in (3.13) and the length of the wetted area $c(t)$. If the shape function $\omega(x, t)$ is assumed given, then the system of integral equations (3.3), (3.17) and (3.18) serves to compute the three unknown functions $\bar{\varphi}_{x}(x), A(x)$ and $c(t)$.

Combining (3.17) and (3.18) we obtain

$$
\int_{0}^{c} \frac{\zeta(u, t)}{\sqrt{u(c-u)}} \mathrm{d} u=\int_{0}^{t} \frac{\Phi_{x}(\xi, 0, t)}{\sqrt{(t-\xi)(t+c-\xi)}} \mathrm{d} \xi,
$$

where the function $\zeta(x, t)$ describes the shape of the elastic plate in the moving coordinate system (see (2.2)) and $\Phi_{x}(x, 0, t)$ is the horizontal displacement of liquid 
particles in the wake behind the plate. Without accounting for the wake, (3.19) was derived by Korobkin (1995).

Equations (3.15), (3.17) and (3.18) specify the asymptotic behaviour of the freesurface elevation at the rear and front contact points. It can be shown that close to the turnover point $x=t+c$ the elevation behaves as

$$
\eta(x, t)=\omega(t+c, t)-\frac{2|B(t)|}{\pi \sqrt{c}(1+\dot{c})} \sqrt{x-t-c}+O\left((x-t-c)^{3 / 2}\right),
$$

where $B(t)$ given by (3.5) is negative (see $\S 3.1$ ) for the regimes under consideration. The free-surface behaviour close to the trailing edge, $x=t$, is given by

$$
\eta(x, t)=\omega(t, t)-\omega_{x}(t, t)(t-x)+\frac{2}{3} H(t)(t-x)^{3 / 2}+O\left((x-t)^{5 / 2}\right),
$$

where

$$
H(t)=-\frac{1}{\pi \sqrt{c}}\left(\frac{1+\dot{c}}{c} B(t)+2 \int_{t}^{t+c} \sqrt{\frac{t+c-\xi}{\xi-t}} \omega_{t t}(\xi, t) \mathrm{d} \xi\right) .
$$

Note that $p(x, 0, t) \sim H(t) \sqrt{x-t}$ where $x \rightarrow t+0$. Therefore if the free surface is convex (as seen from the air side) close to the trailing edge, then the pressure on the plate nearby is negative. A concave free surface implies a positive pressure on the plate nearby.

\subsection{Structural part of the problem}

The function $\zeta(s, t)$ in (2.2) is presented as a linear superposition of normal modes $\psi_{k}(s)$ :

$$
\zeta(s, t)=\sum_{k=0}^{\infty} a_{k}(t) \psi_{k}(s) \quad(0 \leqslant s \leqslant 1)
$$

with the principal coordinates $a_{k}(t)$ to be determined. The normal modes $\psi_{k}(s)$ are the eigensolutions of the boundary-value problem

$$
\begin{gathered}
\psi_{k}^{(\mathrm{iv})}=\lambda_{k}^{4} \psi_{k} \quad(0<s<1), \\
\psi_{k}^{\prime \prime}=\psi_{k}^{\prime \prime \prime}=0 \quad(s=0 \text { and } s=1),
\end{gathered}
$$

where $\lambda_{k}$ are the corresponding eigenvalues. The modes $\psi_{k}(s)$ are the 'dry' modes of a free-free elastic plate. The modes $\psi_{k}(s)$ are orthogonal and in the following the normalized modes are used, such that

$$
\int_{0}^{1} \psi_{k}^{2}(s) \mathrm{d} s=1 \quad(k \geqslant 0) .
$$

The first two modes $\psi_{0}(s)=1$ and $\psi_{1}(s)=\sqrt{3}(2 s-1)$ correspond to zero eigenvalues, $\lambda_{0}=\lambda_{1}=0$. These modes describe the vertical translation and rotation of the rigid plate, respectively. For $k \geqslant 2$ the modes correspond to elastic deformations of the plate. Equations (3.24)-(3.26) have the solution

$$
\begin{aligned}
\psi_{k}(s) & =\cosh \left(\lambda_{k} s\right)+\gamma_{k} \sinh \left(\lambda_{k} s\right)+\cos \left(\lambda_{k} s\right)+\gamma_{k} \sin \left(\lambda_{k} s\right), \\
\gamma_{k} & =-\frac{\cosh \left(\lambda_{k}\right)-\cos \left(\lambda_{k}\right)}{\sinh \left(\lambda_{k}\right)-\sin \left(\lambda_{k}\right)},
\end{aligned}
$$


where $\lambda_{k+1}>\lambda_{k}$ for $k \geqslant 2$ are solutions of the dispersion relation

$$
\cos \left(\lambda_{k}\right) \cosh \left(\lambda_{k}\right)=1 \text {. }
$$

Equation (3.29) implies that $\lambda_{k}$ tends to $\pi(k-1 / 2)$ and $\gamma_{k}$ converges to -1 exponentially as $k \rightarrow \infty$.

Substituting (3.23) in (2.3) and projecting the result onto each individual mode $\psi_{k}(s)$ with the help of (3.24)-(3.26), we obtain a system of ordinary differential equations for the principal coordinates $a_{k}(t)$ :

$$
\mu \ddot{a}_{k}+\theta \lambda_{k}^{4} a_{k}=\int_{0}^{c} p(s+t, 0, t) \psi_{k}(s) \mathrm{d} s-\mu \kappa \delta_{0 k} \quad(k=0,1,2, \ldots) .
$$

Here $\delta_{k l}=1$ for $k=l$ and $\delta_{k l}=0$ for $k \neq l$. The integral in (3.30) is calculated from (3.4)-(3.7) for the hydrodynamic pressure and (3.23) for the shape of the elastic plate. Equation (3.30) is written in matrix-vector form as

$$
\boldsymbol{M}(c) \ddot{\boldsymbol{a}}=\boldsymbol{D}(c, \dot{c}) \dot{\boldsymbol{a}}+\boldsymbol{S}(c, \dot{c}) \boldsymbol{a}+\boldsymbol{d}(c, \dot{c}) \int_{0}^{t} \sqrt{\frac{t-\xi}{t+c-\xi}} \bar{\varphi}_{x}(\xi) \mathrm{d} \xi-\mu \kappa \boldsymbol{e}_{0},
$$

where $\boldsymbol{a}(t)=\left(a_{0}(t), a_{1}(t), a_{2}(t), \ldots\right)^{\mathrm{T}}$ and $\boldsymbol{e}_{0}=(1,0,0, \ldots)^{\mathrm{T}}$. The elements of the matrices $\boldsymbol{M}(c), \boldsymbol{D}(c, \dot{c}), \boldsymbol{S}(c, \dot{c})$, and the vector $\boldsymbol{d}(c, \dot{c})$ are

$$
\begin{aligned}
\boldsymbol{M}_{k l}(c) & =\Lambda_{c}\left(\Psi_{l}, \psi_{k}\right)+\mu \delta_{k l}, \\
\boldsymbol{D}_{k l}(c, \dot{c}) & =2 \Lambda_{c}\left(\psi_{l}, \psi_{k}\right)-\frac{1+\dot{c}}{\pi c} \Gamma_{c}^{(1)}\left(\psi_{k}\right) \Gamma_{c}^{(1)}\left(\psi_{l}\right), \\
\boldsymbol{S}_{k l}(c, \dot{c}) & =-\Lambda_{c}\left(\psi_{l}^{\prime}, \psi_{k}\right)-\theta \lambda_{k}^{4} \delta_{k l}+\frac{1+\dot{c}}{\pi c} \Gamma_{c}^{(1)}\left(\psi_{k}\right) \Gamma_{c}^{(1)}\left(\psi_{l}^{\prime}\right), \\
d_{k}(c, \dot{c}) & =-\frac{1+\dot{c}}{\pi c} \Gamma_{c}^{(1)}\left(\psi_{k}\right),
\end{aligned}
$$

where $\Psi_{k}(s)$ is the $s$-integral of $\psi_{k}(s)$. The functionals $\Lambda_{c}$ and $\Gamma_{c}^{(1)}$ are defined by

$$
\begin{aligned}
\Lambda_{c}\left(f_{1}, f_{2}\right) & =\frac{1}{\pi} \int_{0}^{c} \int_{0}^{c} \sqrt{\frac{v(c-v)}{u(c-u)}} \frac{f_{1}(u) f_{2}(v)}{u-v} \mathrm{~d} u \mathrm{~d} v, \\
\Gamma_{c}^{(1)}(f) & =\int_{0}^{c} \sqrt{\frac{u}{c-u}} f(u) \mathrm{d} u .
\end{aligned}
$$

It can be shown that $\Lambda_{c}\left(\Psi_{l}, \psi_{k}\right)=\Lambda_{c}\left(\Psi_{k}, \psi_{l}\right)$, which implies that the matrix $\boldsymbol{M}$ is symmetric. Note the damping term in (3.31).

It will be shown in $\S 6$ that the only mechanism of extracting energy from the plate vibration is due to the spray jet formed at $x=t+c(t)$, which is the turnover point within the Wagner model of water impact.

\section{Hydroelastic problem}

The hydrodynamic pressure from $\S 3.1$, the flow in the wake from $\S 3.2$, and the motion of the plate from $\S 3.3$ are coupled with the motion of the turnover point $x=t+c(t)$. The equation for $c(t)$ follows from (3.17). Differentiating (3.17) with 
respect to time, we find an ordinary differential equation for $c(t)$ :

$$
\begin{aligned}
& \frac{\mathrm{d} c}{\mathrm{~d} t}=\frac{2 B(t)}{\int_{0}^{t} \frac{\sqrt{t-\xi}}{(t+c-\xi)^{3 / 2}} \Phi_{x}(\xi, 0, t) \mathrm{d} \xi-\left(a_{k}, \frac{1}{c}\left[\Gamma_{c}^{(1)}\left(\psi_{k}\right)+\Gamma_{c}^{(2)}\left(\psi_{k}\right)\right]+2 \Gamma_{c}^{(1)}\left(\psi_{k}^{\prime}\right)\right)}-1, \\
& \Gamma_{c}^{(2)}(f)=\int_{0}^{c} \sqrt{\frac{c-u}{u}} f(u) \mathrm{d} u,
\end{aligned}
$$

where $B(t)$ is defined in (3.5) and is given by

$$
B(t)=\int_{0}^{t} \sqrt{\frac{t-\xi}{t+c-\xi}} \bar{\varphi}_{x}(\xi) \mathrm{d} \xi-\left(a_{k}, \Gamma_{c}^{(1)}\left(\psi_{k}^{\prime}\right)\right)+\left(\dot{a}_{k}, \Gamma_{c}^{(1)}\left(\psi_{k}\right)\right) .
$$

The terms in brackets in (4.1) and (4.3) are inner products, $\left(a_{k}, b_{k}\right)=\sum_{k=0}^{\infty} a_{k} b_{k}$. The integrals in (4.1) and (4.3) describe the influence of the wake on the motion of the turnover point. These integrals are evaluated numerically. The initial conditions for the system of ordinary differential equations (3.31) and (4.1) are

$$
\boldsymbol{a}(0)=\left(\frac{1}{2}, \frac{1}{6} \sqrt{3}, 0,0, \ldots\right)^{\mathrm{T}}, \quad \dot{\boldsymbol{a}}(0)=(-\chi, 0,0,0, \ldots)^{\mathrm{T}}, \quad c(0)=0 .
$$

The functions $\bar{\varphi}_{x}(x)$ and $A(x)$, which are needed to calculate the integrals in (3.31), (4.1) and (4.3), are obtained by solving the integral equations (3.3) and (3.18). A numerical scheme solving the final equations (3.3), (3.18), (3.31), (4.1), (4.4) and evaluating the hydrodynamic pressure is presented in the Appendix.

\section{Oblique impact of a rigid plate at constant velocity}

If the plate is rigid and moves with constant velocity, then we can derive analytical results to start the numerical calculations for the full problem described in the Appendix. The position of the moving plate in (2.1) is given by $\omega(x, t)=x-(1+\chi) t$, where $\chi$ is the non-dimensional vertical velocity of the plate. The resulting flow is described by (2.7)-(2.11) and is self-similar with $\varphi / t$ dependent on the variables $x / t$ and $y / t$. As a result of this self-similarity, the function $\bar{\varphi}_{x}$ (see (2.10)) and the speed of the turnover point $\dot{c}$ are constants with $\bar{\varphi}_{x}(x)=\varphi_{x}^{*}$ and $c(t)=\dot{c}_{*} t$, where the asterisk stands for the constant value of the corresponding function. In terms of the displacement potential (3.8) in the wake region, the condition (3.13) implies that $A(x)=A_{*} x$ and $\Phi_{x}(x, 0, t)=t\left[(1-x / t) \varphi_{x}^{*}+A_{*} x / t\right]$. The latter formula is used to evaluate the integrals in (3.17) and (3.18). Changing the variable of integration in (3.3), (3.17) and (3.18) to $u=\xi / t$ and substituting $\bar{\varphi}_{x}(x)=\varphi_{x}^{*}, \omega(t u, t)=t(u-1-\chi)$ and $\Phi_{x}(t u, 0, t)=t\left[(1-u) \varphi_{x}^{*}+A_{*} u\right]$, we obtain a system of three algebraic equations with respect to three constants $\varphi_{x}^{*}, A_{*}$ and $\dot{c}_{*}$. The solution of this system in parametric form is

$$
\dot{c}_{*}=q^{-2}, \quad \varphi_{x}^{*}=-\frac{\pi}{4 q} \frac{\chi-1+2 q^{2}(1+2 \chi)}{\left(q^{2}+1\right)^{3 / 2}}, \quad A_{*}=-\varphi_{x}^{*} \frac{2 q^{2}-1}{2 q^{2}+2},
$$

where $q(\chi)$ depends on the downward velocity $\chi$ through the following:

$$
\log \left(q+\sqrt{q^{2}+1}\right)=q \sqrt{q^{2}+1} \frac{\chi+3-2 \chi q^{2}}{\chi-1+2(2 \chi+1) q^{2}} .
$$



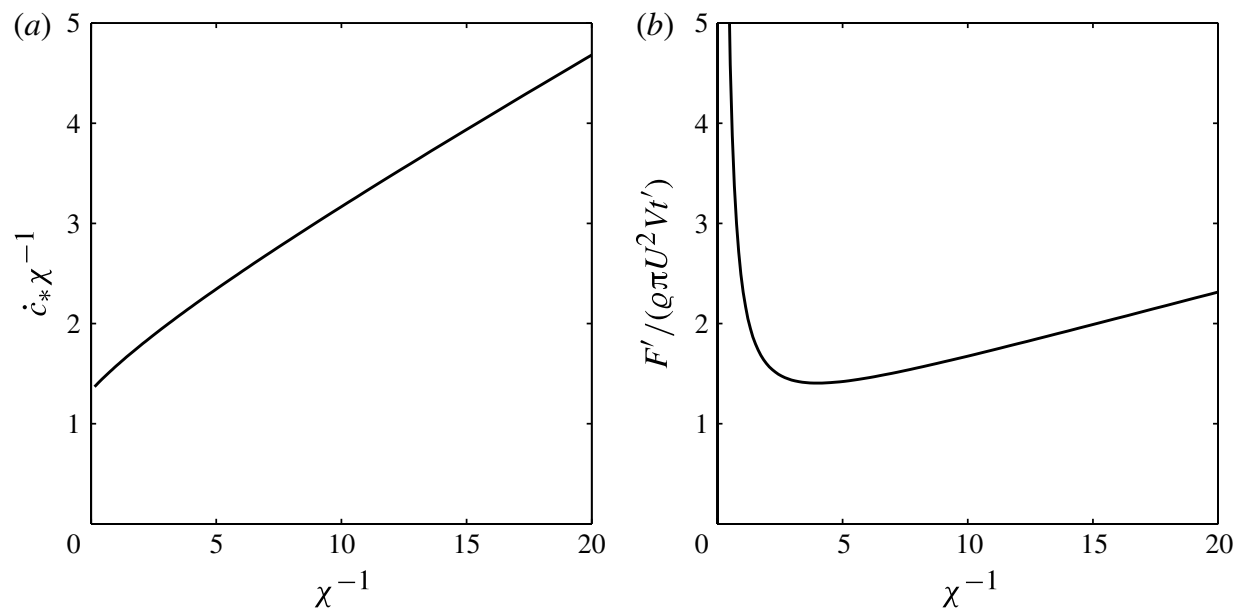

FIGURE 2. Results for a rigid plate that enters the water surface with constant vertical velocity $\chi$. (a) Ratio of the Wagner wetted length to the length of the $x$-interval where the body is below the undisturbed surface, $1 \leqslant x / t \leqslant 1+\chi$, also known as the von Kármán wetted length. $(b)$ The vertical hydrodynamic force acting on the plate.

We have found that $\dot{c}_{*}>2 \chi /(\chi+3)$ for any $\chi>0$, the horizontal velocity $\varphi_{x}^{*}$ in the wake is always negative, and $A_{*}$ can be negative or positive depending on the magnitude of $\chi$.

The pressure distribution along the wetted part of the plate, $t<x<t+\dot{c}_{*} t$, is given by (3.4), where now $T(x, t)=0$ and $B(t)=2 t \varphi_{x}^{*} \sqrt{q^{2}+1} / q$,

$$
p(x, 0, t)=p_{*}(\chi) \sqrt{\frac{x / t-1}{1+\dot{c}_{*}-x / t}}, \quad p_{*}(\chi)=2 \chi+1+\frac{\chi-1}{2 q^{2}(\chi)} .
$$

The vertical component of hydrodynamic force $F(t)$ acting on the plate is

$$
F(t)=\frac{\pi}{2} t p_{*}(\chi) \dot{c}_{*}
$$

The hydrodynamic pressure (5.3) is positive at the wetted part of the plate. The pressure is square-root singular at the turnover point $x=t+\dot{c}_{*} t$ and is zero at the trailing edge.

To demonstrate the importance of water pile-up in front of the plate, we compare the speed of the turnover point $\dot{c}_{*}$ with respect to the plate and the speed of the intersection point, $x-t=\chi t$, between the plate and the initial level of the liquid $y=0$ in figure 2(a), in terms of $\chi^{-1}$. It is seen that the pile-up effect is more pronounced for small vertical speeds of the plate. For large vertical speeds, when $\chi \rightarrow \infty$, we obtain $\dot{c}_{*} / \chi \rightarrow 4 / 3$, agreeing with the position of the contact point for the vertical impact of an inclined plate with zero horizontal speed, where we find $c^{\prime}\left(t^{\prime}\right)=(4 / 3) V t^{\prime} / \alpha$.

The hydrodynamic force $F^{\prime}$ is a linear function of time $t^{\prime}$ in this problem. The force is scaled in the same way as in Ulstein \& Faltinsen (1996) and Sedov (1940). Our calculations yield

$$
\frac{F^{\prime}}{\varrho_{F} U^{2} V t^{\prime} \pi}=\frac{\dot{c}_{*} p_{*}(\chi)}{2 \chi} .
$$


The non-dimensional force (5.5) is shown as a function of $\chi^{-1}$ in figure $2(b)$. For large vertical speed, $\chi \rightarrow \infty$, we obtain

$$
\frac{\dot{c}_{*} p_{*}(\chi)}{2 \chi} \sim \frac{4}{9} \chi^{2} .
$$

The solution obtained will be used below to start the computations of the unsteady motion of either a rigid or an elastic plate, governed by either Newton's laws or Euler's equation (2.3).

\section{An account of work and energy}

In this section we identify the energies contributing to the fluid-plate system. Although we neglect the jet in the hydrodynamic model, the energy in the jet contributes significantly to the total energy. Nonlinear terms in Euler's equation of fluid flow become significant in the jet-root region, since the fluid velocity is of order $U$ there, hence the linearized hydrodynamic model breaks down in the jet-root region.

First, to find the energy in the jet explicitly, we need to know the flow in the jet-root region. During the early stage of impact the flow in the jet root can be approximated by the quasi-steady Kelvin-Helmholtz cavity flow as shown in Howison et al. (1991). We introduce the inner variables $\tilde{x}=(x-t-c) / \alpha^{2}, \tilde{y}=(y-\alpha \zeta(c, t)) / \alpha^{2}$ and inner velocity potential $\tilde{\varphi}(\tilde{x}, \tilde{y})$ proportional to $\alpha^{-1} \varphi(x, y) /(1+\dot{c})-\tilde{x}$. Then the complex potential of the nonlinear flow is given by (Birkhoff \& Zarantonello 1957)

$$
\tilde{\varphi}+\mathrm{i} \tilde{\psi}=-\frac{\tilde{\delta}}{\pi}\left(\frac{4 \tilde{f}}{(\tilde{f}+1)^{2}}+2 \log \left(\frac{\tilde{f}-1}{\tilde{f}+1}\right)\right)
$$

where $\tilde{\psi}$ is the stream function, $\tilde{f}=\tilde{\varphi}_{\tilde{x}}-\mathrm{i} \tilde{\varphi}_{\tilde{y}}$ is the complex velocity and $\tilde{\delta}$ is the thickness of the jet corresponding to the length scale of the inner region. It follows from (6.1) that the horizontal velocity along the plate in the far field of the inner region, $\tilde{x} \rightarrow-\infty, \tilde{y}=0$, is

$$
\tilde{\varphi}_{\tilde{x}}(\tilde{x}, 0)=-1+\sqrt{-\frac{4 \tilde{\delta}}{\pi \tilde{x}}}+O\left((-\tilde{x})^{-3 / 2}\right)
$$

which is matched with the outer horizontal flow velocity in the region $x=t+c+O(\alpha)$ given by (3.1) to obtain the thickness of the jet

$$
\delta=\frac{\alpha^{2} B^{2}(t)}{4 \pi c(1+\dot{c})^{2}}
$$

in $x O y$ where $\delta=\alpha^{2} \tilde{\delta}$. The non-dimensional jet velocity in the reference frame moving with the turnover region is $1+\dot{c}$, so the jet velocity in the global frame of reference $x O y$ is $2(1+\dot{c})$, hence the energy flux into the jet in non-dimensional variables is (see Cointe et al. 2004)

$$
\frac{\mathrm{d}}{\mathrm{d} t} E_{j e t}^{(f)}=\frac{1}{2}[2(1+\dot{c})]^{2} \delta(1+\dot{c}) \frac{1}{\alpha^{2}}=\frac{1+\dot{c}}{2 \pi c} B^{2}(t),
$$

where we have scaled the energies by $\alpha^{2} \varrho_{F} U^{2} L^{2}$. Secondly, the rate of change of the kinetic energy of the fluid bulk (excluding that in the jet) can be written, using Green's 
first identity and (2.7) and (2.9),

$$
\begin{aligned}
\frac{\mathrm{d}}{\mathrm{d} t} E_{k i n}^{(f)} & =\frac{1}{2} \frac{\mathrm{d}}{\mathrm{d} t} \int_{\{y \leqslant 0\}}\left(\varphi_{x}^{2}+\varphi_{y}^{2}\right) \mathrm{d} x \mathrm{~d} y=\left.\frac{1}{2} \frac{\mathrm{d}}{\mathrm{d} t} \int_{0}^{t+c} \varphi \varphi_{y}\right|_{y=0} \mathrm{~d} x \\
& =\frac{1}{2} \int_{t}^{t+c} \varphi_{t} \varphi_{y} \mathrm{~d} x+\left.\frac{1}{2} \int_{0}^{t+c} \varphi \varphi_{t y}\right|_{y=0} \mathrm{~d} x .
\end{aligned}
$$

It can be shown with the help of Green's second identity and (2.6) and (2.8) that

$$
\frac{\mathrm{d}}{\mathrm{d} t} E_{k i n}^{(f)}=-\int_{t}^{t+c} p \omega_{t} \mathrm{~d} x-\frac{1+\dot{c}}{2 \pi c} B^{2}(t),
$$

where $p=p(x, 0, t)$ in this section. Equations (6.4) and (6.6) give

$$
\frac{\mathrm{d}}{\mathrm{d} t}\left(E_{k i n}^{(f)}+E_{j e t}^{(f)}\right)=-\int_{t}^{t+c} p \omega_{t} \mathrm{~d} x
$$

where the right-hand side is the time-derivative of the work done by the elastic plate on the fluid and $E_{\text {kin }}^{(f)}+E_{\text {jet }}^{(f)}$ is the total kinetic energy of the flow both in the bulk and in the jet. Thirdly, Euler's beam equation (2.3) implies

$$
\frac{\mathrm{d}}{\mathrm{d} t}\left(E_{\text {kin }}^{(p)}+E_{\text {bend }}^{(p)}+E_{\text {grav }}^{(p)}\right)=\int_{t}^{t+c} p\left(\omega_{x}+\omega_{t}\right) \mathrm{d} x,
$$

where the kinetic energy, the elastic potential energy and the gravitational potential energy of the plate are

$$
E_{\text {kin }}^{(p)}=\frac{\mu}{2} \int_{0}^{1} \zeta_{t}^{2} \mathrm{~d} s, \quad E_{\text {bend }}^{(p)}=\frac{\theta}{2} \int_{0}^{1} \zeta_{s s}^{2} \mathrm{~d} s, \quad E_{\text {grav }}^{(p)}=\mu \kappa \int_{0}^{1} \zeta \mathrm{d} s,
$$

respectively. Now, to keep the horizontal velocity component of the plate constant, the plate does work $W(t)$ on the fluid. The time-derivative of this work in non-dimensional variables is

$$
\frac{\mathrm{d}}{\mathrm{d} t} W=\int_{t}^{t+c} p \omega_{x} \mathrm{~d} x
$$

By combining (6.7), (6.8) and (6.10) we find

$$
\frac{\mathrm{d}}{\mathrm{d} t}\left(E_{\text {kin }}^{(p)}+E_{\text {bend }}^{(p)}+E_{\text {grav }}^{(p)}+E_{\text {kin }}^{(f)}+E_{\text {jet }}^{(f)}-W\right)=0 .
$$

Equation (6.11) implies that the total energy of the plate-liquid system including the jet energy is equal to the work $W(t)$ done on the fluid at any time instant during impact. The components of the total energy can be of different magnitude. It is valuable to investigate where the energy supplied to the plate-liquid system goes. It will be shown in $\S 7$ that a main part of this energy leaves the flow region with the jet. The energy conservation law in (6.11) is also used to verify the accuracy of our numerical results.

\section{Numerical results}

There are two non-dimensional parameters, $\chi=V /(U \alpha)$ and $\mu$, which govern the rigid-plate impact, when the gravity force is neglected compared to the hydrodynamic force acting on the plate. Here $\mu$ is proportional to the mass of the plate divided by its 

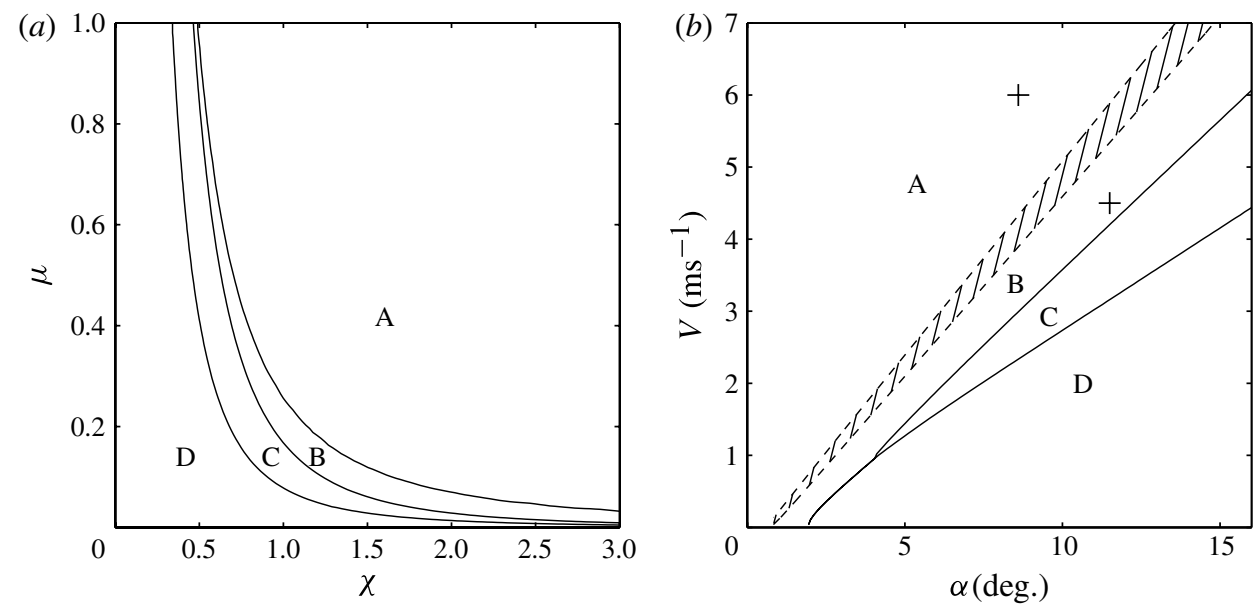

Figure 3. (a) Plane of the parameters $\chi, \mu$ for the rigid-plate impact without gravity, and (b) plane of the initial vertical speed $V$ and initial inclination angle $\alpha$ for the elastic-steel-plate impact with gravity. There are four scenarios of oblique impact of the plate, sketched in figure 4: A, the plate is completely wetted at the end of the calculations; B, an air cavity is trapped close to the leading edge of the plate; $\mathrm{C}$, wake regions appear on both sides of the wetted area of the plate; $\mathrm{D}$, the plate exits the water before being completely wetted. Classes $\mathrm{A}, \mathrm{B}$ and $\mathrm{C}$ are also found to occur in the shaded region of figure $3(b)$. The points marked by + correspond to Cases 1 and 2 described in the main text.

added mass (see $\S 2.1$ ). The parameter plane $(\chi, \mu)$ can be divided into four parts, as shown in figure 3(a). The corresponding sketches of the flow configurations are shown in figure 4. To determine the regions corresponding to the different outcomes of the plate impact, the calculations were performed for $\chi$ from 0.3 to 3 with step 0.03 , and for $\mu$ from 0.01 to 1 with step 0.01 . The precise dividing curves have been computed by a walk along each boundary. We successively determined the points along the dividing curves with a fixed distance from the previous point by the bisection method. For impact conditions from region $\mathrm{A}$, the plate is completely wetted at the end of the impact, with the speed of the Wagner contact point, $1+\dot{c}(t)$, being positive from the beginning to the end of the impact stage. In region $\mathrm{B}$, the leading edge of the rigid plate enters the disturbed liquid free surface before the Wagner contact point arrives at the leading edge. Despite the high forward speed, this scenario is similar to the so-called blockage phenomenon discussed in Korobkin \& Khabakhpasheva (2006) for an elastic plate entering the water vertically. In region $\mathrm{C}$, the speed of the Wagner contact point in the global coordinate system, $1+\dot{c}$, becomes negative well before the plate is completely wetted, so that the free surface at the forward contact point starts to separate from the plate. It was discovered that in regions $\mathrm{B}$ and $\mathrm{C}$ the trailing edge of the plate is above the initial water surface and the current inclination angle of the plate is negative before the present model becomes invalid. In both classes, B and $\mathrm{C}$, the present model could be modified to accommodate the discovered phenomena shown in figure 4 . In class B, an air cavity is captured under the plate and our model may be extended to account for its presence (see Korobkin \& Khabakhpasheva 2006, section 5 for some discussions). In class $\mathrm{C}$, a wake region can be introduced to replace the Wagner contact point at the time instant when $\dot{c}=-1$ (see Reinhard, Korobkin \& Cooker 2012a). The impact conditions from region D provide an exit of the plate 


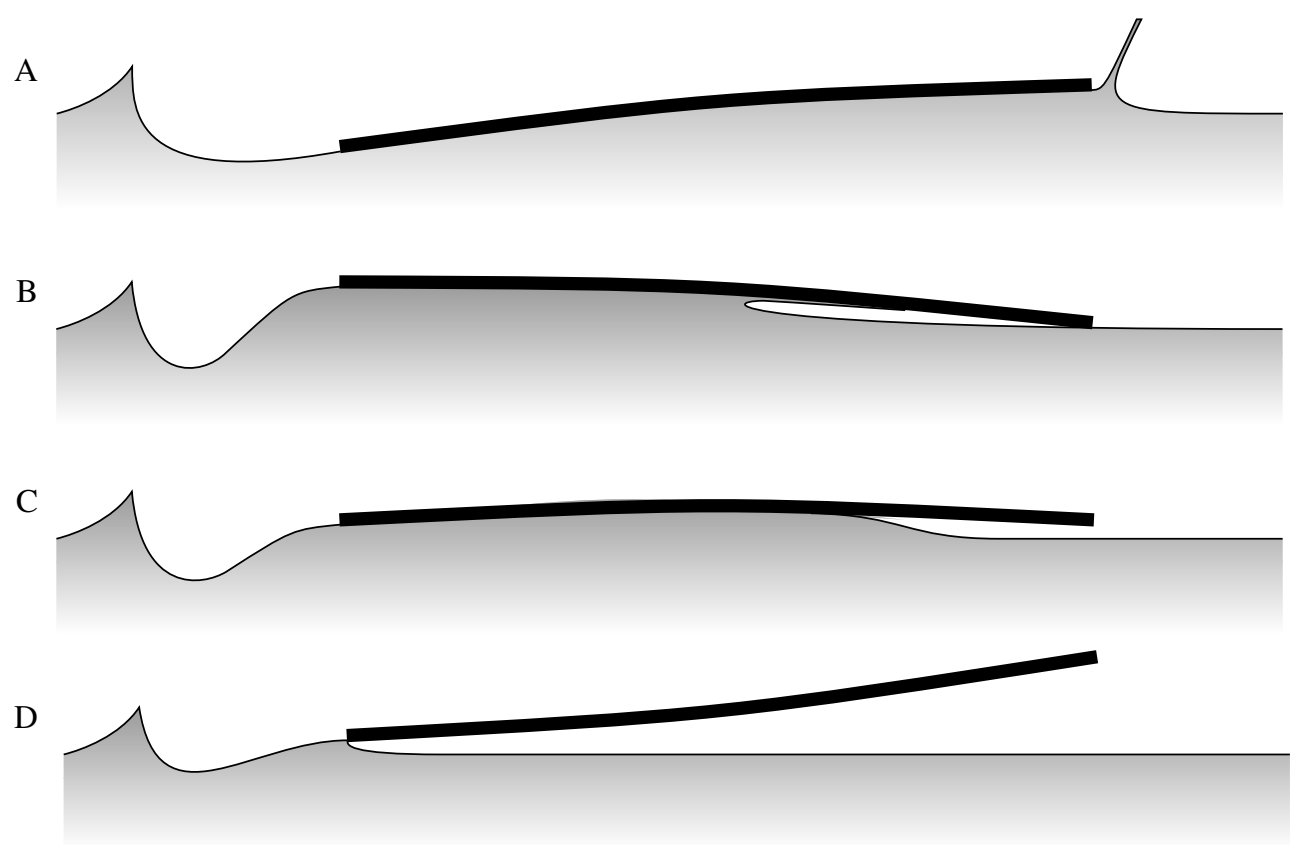

FIGURE 4. Sketches of the four flow configurations at the end of computations for an elastic plate within the present model. Classes A, B, C, D are described in the main text and in the caption for figure $3(a, b)$. Computations terminate for the following reasons: for class $\mathrm{A}$ when $c=1$, for class B when $\eta(1+t, t)=\omega(1+t, t)$, for class $\mathrm{C}$ when $\dot{c}=-1$, and for class $\mathrm{D}$ when $c=0$.

from the liquid. In this class the wetted length of the plate increases initially but shrinks later on, with $c(t)$ decreasing to zero while $1+\dot{c}(t)$ remains positive from the beginning to the end of the interaction process. Apart from the scenarios A, B, $\mathrm{C}$ and $\mathrm{D}$ no other types were distinguished in our exploration of the $(\chi, \mu)$-plane in figure 3(a). In particular, values of $\chi$ and $\mu$ which lead to steady planing of the elastic plate were not detected. This is consistent with the work of Hicks \& Smith (2011) for plate impact into shallow water. They too did not find conditions in their parameter space which correspond to flows that lead to steady planing. The flow regimes A and D were also observed in Hicks \& Smith (2011). The flow regimes B and C are new. Their regions are narrow and they require non-trivial extensions of the present model. Such extensions are not further discussed in this paper.

If we include the elasticity of the plate and gravity, the problem depends on four parameters $\chi, \mu, \theta$ and $\kappa$. We are concerned with conditions when the elasticity of the plate matters and the structural behaviour of the plate is strongly coupled with the flow generated by the impact. We found that the time of interaction between a light plate and the fluid in oblique impact is so short that the elasticity of the plate is barely noticed. At the end of the short interaction stage for a light plate, the plate leaves the water by bouncing back up. The hydroelastic effect is much more pronounced for relatively heavy thick plates, when the interaction stage is longer. In our calculations detailed in this section the plate length $L$ is $2.4 \mathrm{~m}$ with plate thickness $54 \mathrm{~mm}$. The plate is made of steel with density $\varrho_{S}=7850 \mathrm{~kg} \mathrm{~m}^{-3}$ and 
flexural rigidity $D=2860 \mathrm{kN} \mathrm{m}$. The horizontal component of the plate velocity was fixed as $U=24 \mathrm{~m} \mathrm{~s}^{-1}$. The gravity acceleration $g$ is $9.81 \mathrm{~m} \mathrm{~s}^{-2}$ in the calculations. The mass of the plate per unit width is rather high, $1017.36 \mathrm{~kg} \mathrm{~m}^{-1}$, which guarantees that the interaction time is long enough for the elastic effects to be pronounced. The plate rigidity is big, however, the hydrodynamic forces acting on the plate were found to be large and strongly dependent on the plate flexibility in the calculations. Note that this configuration implies $\mu=0.18$ and $\theta=0.36$. The only parameters which have not been fixed yet are the vertical component of the initial velocity of the plate, $V$, and the initial angle of attack $\alpha$.

In figure $3(b)$ the distinguished regions $\mathrm{A}, \mathrm{B}, \mathrm{C}$ and $\mathrm{D}$ are shown in the $(\alpha, V)$-plane for the parameter configuration introduced above. In contrast to figure $3(a)$ the plate is elastic and gravity is taken into account in the equations of the plate motion. The deflections of the elastic plate with free ends are of highest amplitude at the end points of the plate. If the contact point comes close to the leading edge at the instant when the current inclination angle of the plate is negative, then the class of the impact strongly depends on the current deflection of the leading edge of the plate. This makes the boundary between regions $\mathrm{A}$ and $\mathrm{B}$ less certain. We identified a region, shaded in figure $3(b)$, in which we found examples of classes $\mathrm{A}, \mathrm{B}$ and even $\mathrm{C}$. The boundaries between $\mathrm{B}, \mathrm{C}$ and between $\mathrm{C}, \mathrm{D}$ are still sharp for a flexible plate. The regions A-D for the rigid plate have almost the same boundaries as in figure $3(b)$, except for the shaded area, which becomes narrower as the plate rigidity increases, and it becomes sharp when the plate is rigid.

Two cases were selected to illustrate the elastic-plate motion with initial conditions from region $\mathrm{A}$ (Case $1, \alpha=8.6^{\circ}, V=6 \mathrm{~m} \mathrm{~s}^{-1}$ ) and region $\mathrm{B}$ (Case 2, $\alpha=11.5^{\circ}$, $V=4.5 \mathrm{~m} \mathrm{~s}^{-1}$ ) in figure $3(b)$. Calculations were performed with five elastic modes and an integration time step $\Delta t=5 \times 10^{-4}$ (see Appendix). The non-dimensional period of the fifth mode is 0.01 , which is 20 times larger than $\Delta t$. It was verified numerically that the relative accuracy of the solution with five modes is better than $1 \%$ for Case 1 . The numerical solutions were compared with those for rigid plates.

The positions of the plate and the free surface at several times are shown in figures 5 and 6 for Case 1 and Case 2, respectively. For the rigid-plate solutions, we only show the positions of the free surfaces behind the plate. Note that the positions of the plate and the free-surface elevations in front of the moving plate are weakly dependent on the plate elasticity. However, the free-surface shape in the wake behind the plate is smooth for the rigid plate but visibly reproduces the vibrations of the elastic plate. The free-surface elevation at the origin corresponding to the splash region is logarithmic singular. To find the correct free-surface contour in the splash region, nonlinear terms in the boundary conditions have to be accounted for (see Faltinsen \& Semenov 2008). For the initial conditions from region B (figure 6) the leading edge of the plate collides with the free surface when less than a third of the plate is wetted $\left(c^{\prime}=0.75 \mathrm{~m}\right.$ at $\left.t^{\prime}=70 \mathrm{~ms}\right)$. A thin air bubble is captured under the plate at this instant.

The motion of the contact points for elastic and rigid plates in Cases 1 and 2 is shown in figure 7(a). The initial inclination angle $\alpha$ is smaller in Case 1, which leads to faster motion of the contact point than in Case 2. The contact point speed is more sensitive to the plate bending in Case 1. Elasticity of the plate may increase the speed of the contact point and, correspondingly, may increase the hydrodynamic loads. The speed of the contact point is rather low in Case 2, which makes the motion of rigid and elastic plates almost identical. 


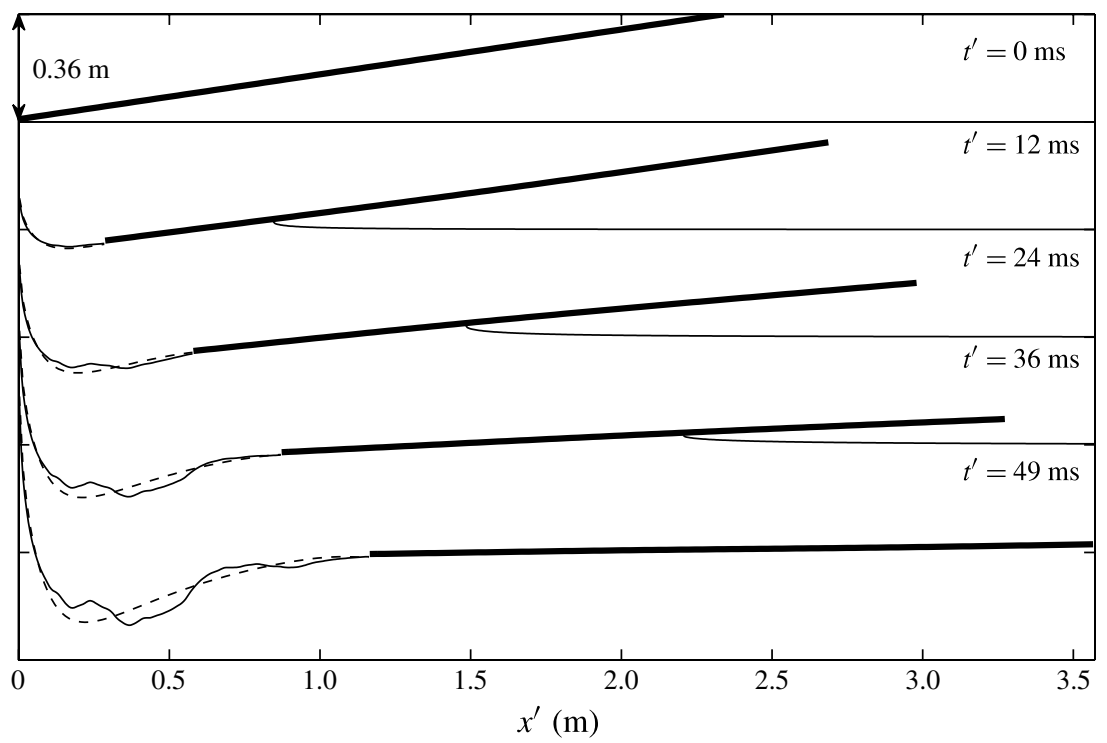

FIGURE 5. Time-evolution of elastic-plate position (thick line), free-surface elevation (thin solid line), and the free-surface elevation for a corresponding rigid plate (thin dashed line) for Case 1 . The vertical scale is the same as the horizontal scale.

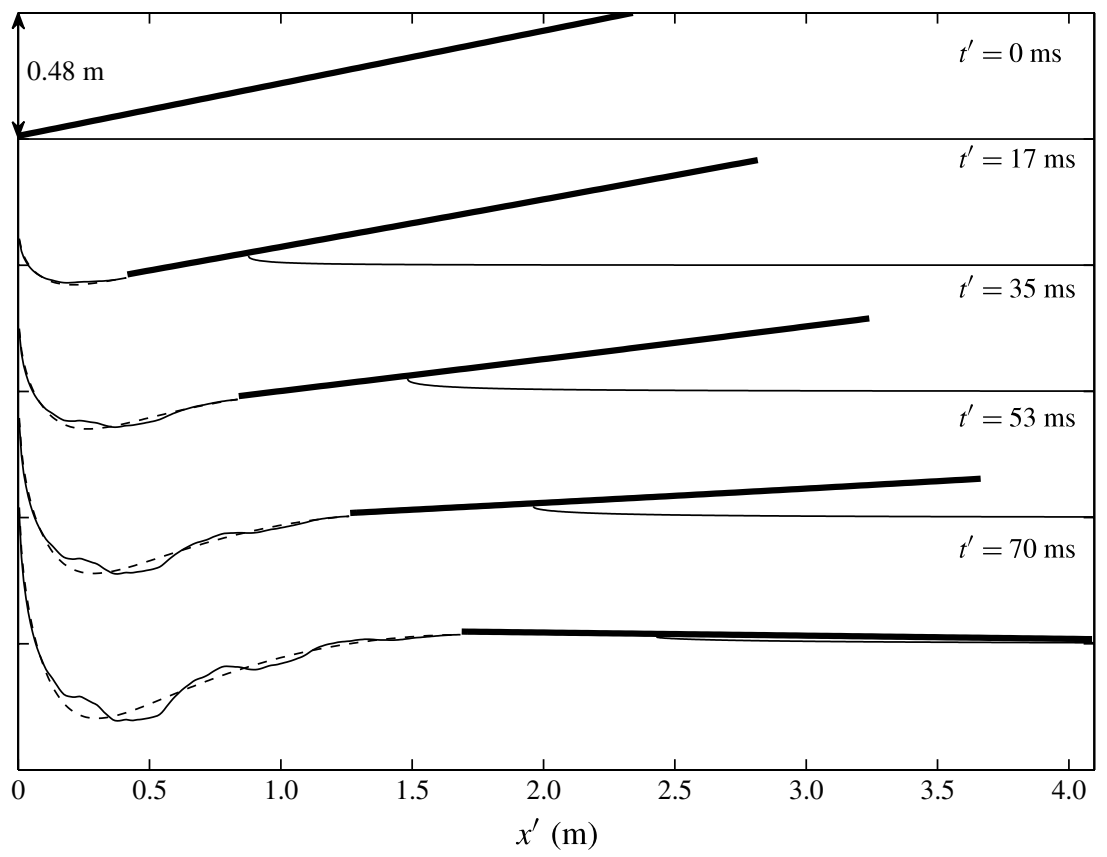

FIGURE 6. Time-evolution of elastic plate position (thick line), free-surface elevation (thin solid line), and the free-surface elevation for a corresponding rigid plate (thin dashed line) for Case 2. The vertical scale is the same as the horizontal scale. 

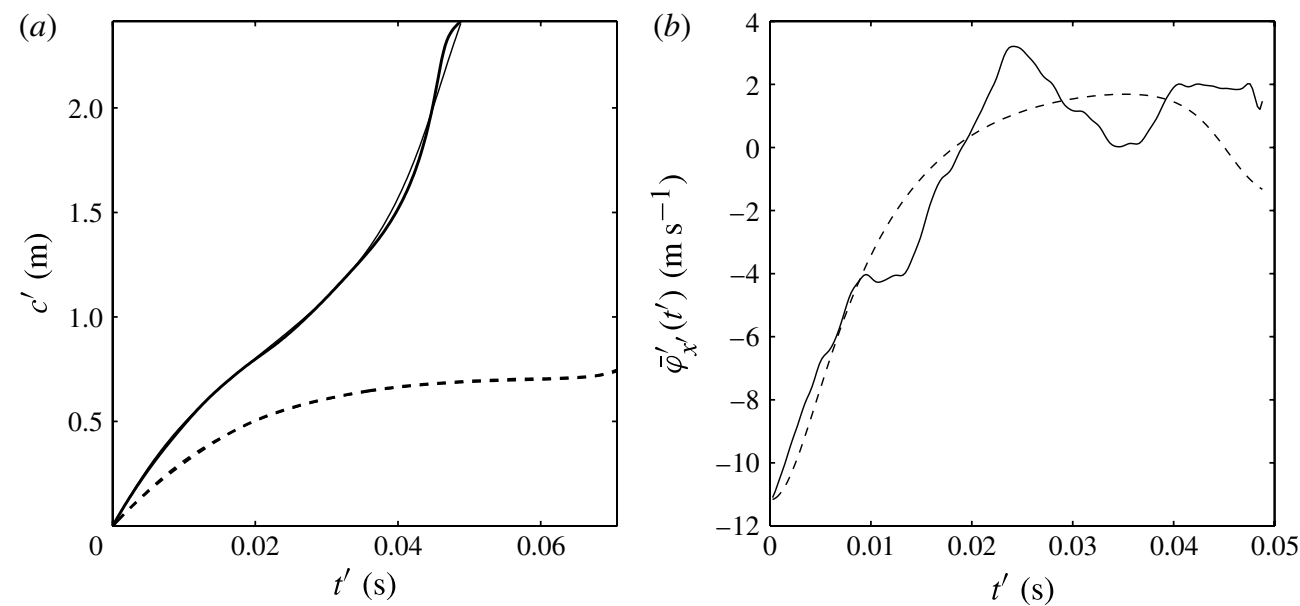

Figure 7. (a) Contact-point motion for Case 1 (solid lines) and Case 2 (overlapping dashed lines) for elastic (thick lines) and rigid (thin lines) plate. (b) Horizontal fluid velocity $\bar{\varphi}_{x^{\prime}}^{\prime}\left(t^{\prime}\right)$ at the trailing edge in Case 1 shown for elastic (solid line) and rigid (dashed line) plate.

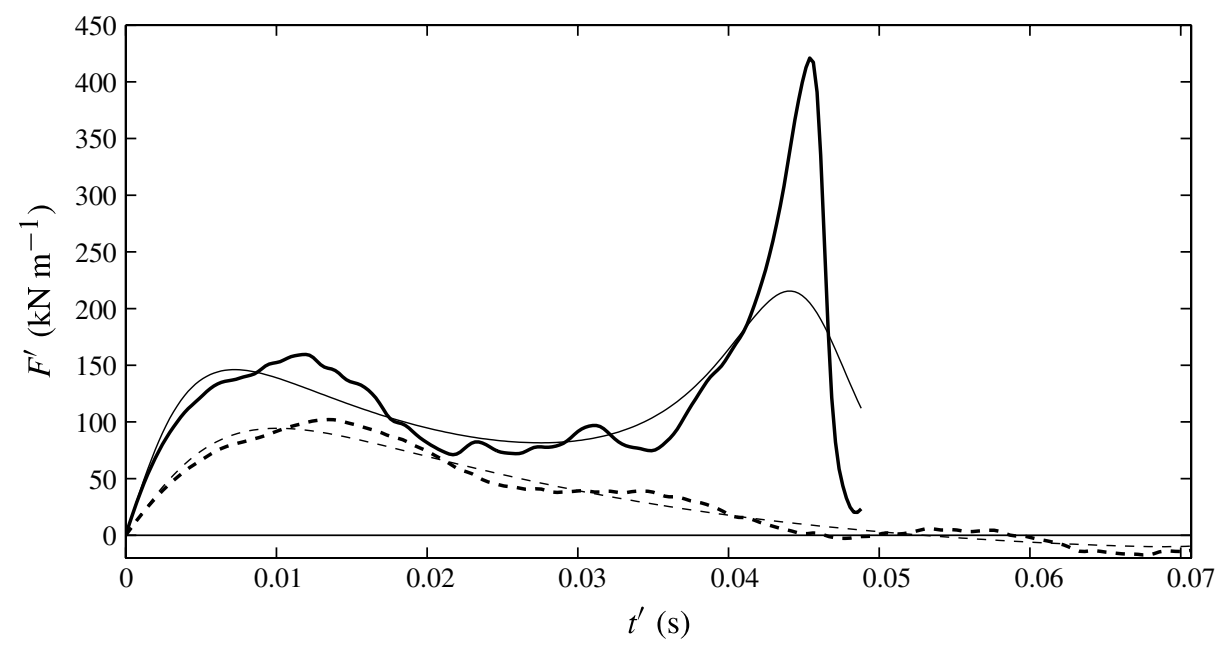

FIGURE 8. Vertical force evolution for elastic-plate (thick lines) and rigid-plate (thin lines) impact for Case 1 (solid lines) and for Case 2 (dashed lines).

The horizontal component of velocity at the trailing edge, $\bar{\varphi}_{x^{\prime}}^{\prime}\left(t^{\prime}\right)$, is plotted as a function of time in figure $7(b)$ for Case 1 . This velocity is rather different for elastic and rigid plates, with corresponding differences in the free-surface elevation (see figure 5). At the beginning the horizontal flow velocity is negative, but then changes to positive values, which continue to be much smaller than the horizontal plate velocity.

Figure 8 shows the vertical hydrodynamic forces acting on rigid and elastic plates in Cases 1 and 2. The forces on elastic and rigid plates in Case 1 are different in amplitude but similar in behaviour. In Case 1, the force has two local maxima. The first maximum is due to the initial increase of the wetted area of the plate. This leads 

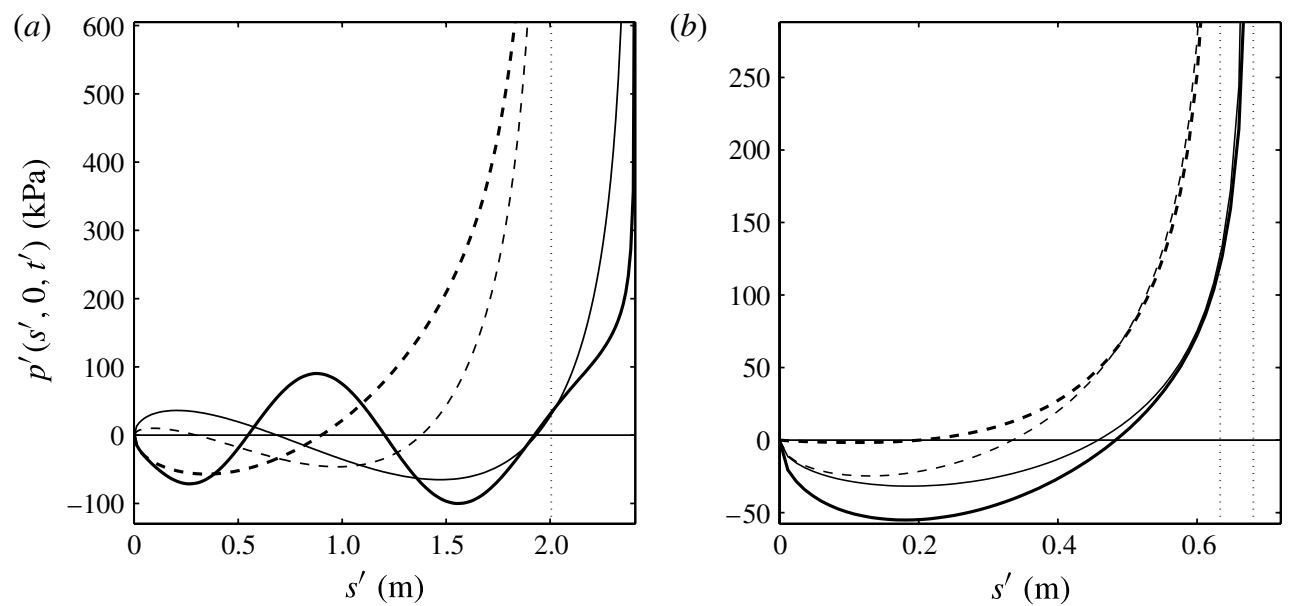

FIGURE 9. Pressure distribution along the plate at $(a) t^{\prime}=45 \mathrm{~ms}$ (dashed lines) and $t^{\prime}=49 \mathrm{~ms}$ (solid lines) for Case 1 , and $(b) t^{\prime}=34 \mathrm{~ms}$ (dashed lines) and $t^{\prime}=45 \mathrm{~ms}$ (solid lines) for Case 2. Rigid-plate (thin lines) and elastic-plate impact (thick lines).

to strong deceleration of the vertical motion and a decrease in the inclination angle of the plate. By the time of the second peak, the angle is rather small but the vertical speed of the leading edge of the plate is large. This forces the contact point to move faster towards the leading edge, increasing quickly the wetted area of the plate and the hydrodynamic force acting on it. Note that the maximum hydrodynamic force for the elastic plate is $415 \mathrm{kN} \mathrm{m}^{-1}$, which is double the corresponding force for the rigid plate. This difference in the force amplitude is explained by bending of the elastic plate towards the liquid and, as a result, a higher speed of the contact point. Just after the second peak the force drops sharply to zero with the plate being completely wetted at the end of the computations. For Case 2, the difference between the forces acting on rigid and elastic plates is also pronounced. The force has a single maximum, which is smaller than in Case 1. At the end of our calculations, the forces become negative in Case 2, which indicates that the hydrodynamic pressure in the wetted area takes negative values.

Figure $9(a)$ shows the pressure distributions for Case 1 at those times when the vertical force (see figure 8$)$ reaches its second maximum $\left(c^{\prime}(45 \mathrm{~ms}) \approx 2.1 \mathrm{~m}\right.$ for rigid and elastic plate) and when the plate is fully wetted $\left(c^{\prime}(49 \mathrm{~ms}) \approx 2.4 \mathrm{~m}\right.$ for the rigid and elastic plate). Corresponding curves for Case 2 are shown in figure $9(b)$ for $t^{\prime}=34 \mathrm{~ms}$ and $t^{\prime}=45 \mathrm{~ms}$. In Case 1 , the pressure in the wetted area oscillates for the elastic plate but is rather uniform for the rigid plate. The negative pressures are more pronounced for elastic plates in both Cases. The fluid may cavitate in the zones of negative pressures, and this is more likely for elastic plates than for rigid ones.

The horizontal component $F_{x}(t)$ of the hydrodynamic force acting on the plate can be estimated as the normal force component given by (A 12) multiplied by the instantaneous angle of plate inclination. The mean value of this force is estimated as $9.9 \mathrm{kN} \mathrm{m}^{-1}$ for Case 1. Viscous drag is less important in our problem. The viscous force $F_{v}$ acting on the rigid surface was estimated by Faltinsen (2005) as $F_{v}=(1 / 2) C_{F} \varrho_{F} \bar{c}^{\prime} U^{2}$, where $C_{F} \approx 0.003$ is the friction coefficient for a flat plate parallel to the uniform flow at speed $U$ and $\bar{c}^{\prime}$ is the mean length of the wetted area of the plate for Case 1 , which is $1 \mathrm{~m}$. The estimate by Faltinsen gives $F_{v}=0.87 \mathrm{kN} \mathrm{m}^{-1}$. 


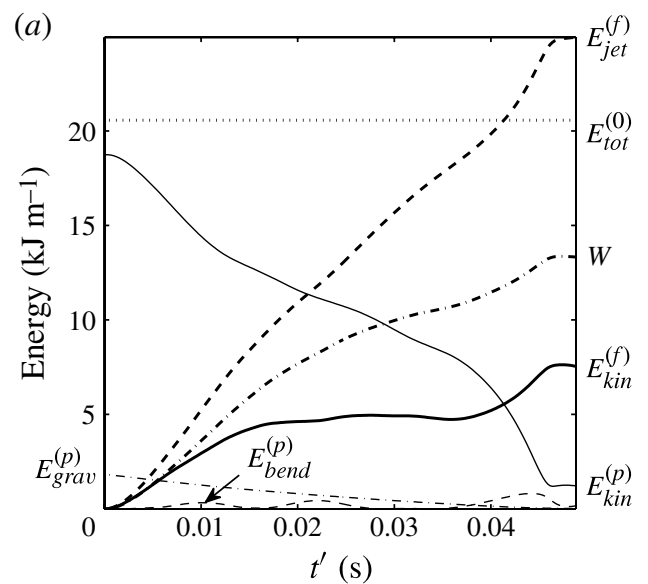

(b)

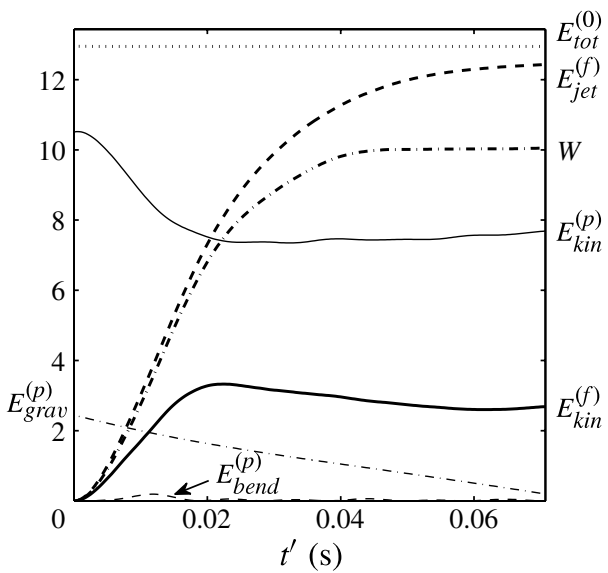

FIGURE 10. Thin lines show $E_{\text {kin }}^{(p)}$ (solid), $E_{\text {bend }}^{(p)}$ (dashed), $E_{\text {grav }}^{(p)}$ (dash-dotted), and thick lines show $E_{\text {kin }}^{(f)}$ (solid), $E_{\text {jet }}^{(f)}$ (dashed), $W$ (dash-dotted), for $(a)$ Case 1 and $(b)$ Case 2.

Note that the viscous force provided by Rayleigh's self-similar solution (see Acheson 1990) for an infinitely long plate moving suddenly with constant velocity, was found even smaller than the estimate by Faltinsen (2005). It follows that the inviscid force $F_{x}(t)$ is much greater than the viscous contribution. In order to keep the horizontal speed of the plate constant, the plate must do some work against this horizontal drag and this work is $W(t)$ as defined in (6.10).

Figure 10 shows how the total energy of the plate-liquid system is partitioned into the kinetic energy of the plate $E_{\text {kin }}^{(p)}(t)$, elastic potential energy of the plate $E_{b e n d}^{(p)}(t)$, gravitational potential energy of the plate $E_{\text {grav }}^{(p)}(t)$, kinetic energy of the flow in the main region $E_{\text {kin }}^{(f)}(t)$, kinetic energy in the spray jet $E_{j e t}^{(f)}(t)$ and the work $W(t)$ done by the plate on the fluid to keep the horizontal velocity constant. Note that $E_{k i n}^{(p)}(t)$ includes the energies of vertical motion and rotation of the plate, as well as the energy of the elastic deflection. In both Cases, the work $W(t)$ is an important component in the energy balance. Figure $10(a, b)$ indicates that the energy supplied to the system mainly goes to the jet. In Case 1 the kinetic energy of the plate almost decays to zero whereas in Case 2 the plate keeps a large part of its initial energy due to its rotation. A minor part is transferred into the kinetic energy of the bulk water flow. The contribution of the plate-bending energy to the energy balance is negligible. The energies presented in figure $10(a, b)$ are much lower than the kinetic energy of the plate due to its horizontal motion, $E_{k i n}^{(h o r)}=318 \mathrm{~kJ} \mathrm{~m}^{-1}$. The latter energy is independent of time $t$. If the horizontal motion is free and determined by Newton's second law, then $E_{\text {kin }}^{(\text {hor }}$ will be reduced by $W$, which is less than $15 \mathrm{~kJ} \mathrm{~m}^{-1}$ in both Cases. This implies that the plate will only slightly decelerate in the horizontal direction and the results of the presented analysis will not change significantly.

The distribution of the bending stresses $\sigma^{\prime}\left(s^{\prime}, t^{\prime}\right)$ in the plate and the maximum stress $\sigma_{\text {max }}^{\prime}\left(t^{\prime}\right)$ are important characteristics of the elastic-plate impact problem. The dimensional stresses $\sigma^{\prime}$ are defined by $\sigma^{\prime}=(E \alpha h / 2 L) \zeta_{s s}$, where $\zeta(s, t)$ is the nondimensional deflection of the plate during the impact. The maximum stress is 

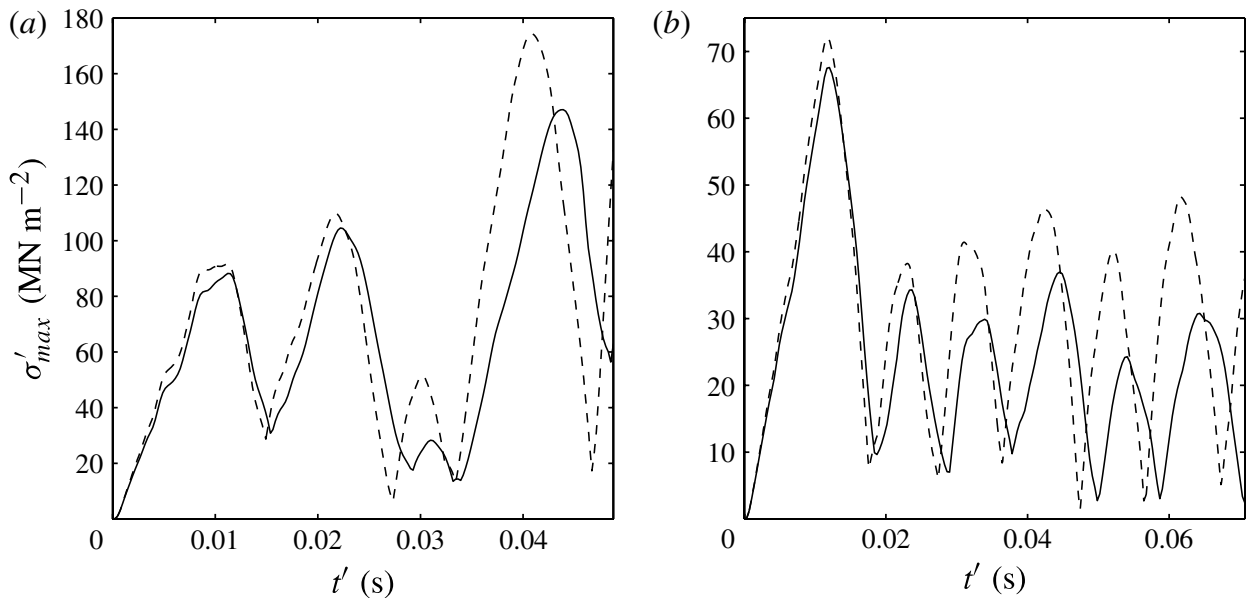

FIGURE 11. Maximum bending stress for the coupled problem (solid line) and for the uncoupled problem (dashed line) as function of time, $(a)$ for Case 1 and $(b)$ for Case 2.

calculated at each time instant $t^{\prime}$ as

$$
\sigma_{\text {max }}^{\prime}\left(t^{\prime}\right)=\max _{0<s^{\prime}<L}\left|\sigma^{\prime}\left(s^{\prime}, t^{\prime}\right)\right| .
$$

Figure 11 shows the time-evolutions of maximum bending stresses for Cases 1 and 2 . Dashed lines in this figure correspond to maximum stress estimated by the decoupled problem of plate impact. In the uncoupled problem we apply the pressure, determined in the problem of the rigid-plate impact, to the elastic plate. There is no elastic interaction between the plate and the fluid. However, the rigid motion of the plate are determined together with the fluid flow. In both Cases the bending stresses calculated by the coupled and decoupled approaches are in good agreement at the beginning, but later differ strongly. For Case 1 the maximum bending stress is comparable with the yield stress which is $\sim 250 \mathrm{MN} \mathrm{m}^{-2}$ for $\mathrm{A} 36$ steel, at the end of the impact stage. In Case 2, see figure $11(b)$, the maximum stress reaches its maximum when the plate bends for the first time. Due to low hydrodynamic forces later, the maximum bending stress decays thereafter. It is possible that the stresses will increase again when the leading edge of the plate collides with the water free surface. The place on the plate where the maximum bending stress occurs can be quite far from the mid-point: our calculations revealed that it is close to the contact point where the pressure is maximal.

\section{Conclusions and further work}

We have developed a fully coupled model of elastic-plate impact onto the free surface of an incompressible fluid, suitable for conditions when the plate has a constant and relatively high forward velocity component. The irrotational flow model includes a wake region behind the plate and a jet region on the front part of the plate. Both rigid-body motion and elastic motion of the plate are determined simultaneously with the hydrodynamic pressure distribution, the shape of the free surface, and the size of the wetted part of the plate. The structural part of the problem is solved by the normal-mode method; the hydrodynamic part of the solution provides an explicit formula for the pressure along the wetted part of the vibrating plate. To determine the 
length of the wetted part we assumed that the spray jet in front of the advancing plate is negligible. All parts of the problem are coupled.

We have identified four different regimes of plate motion, which depend on the impact conditions. At the end of the impact stage analysed we can make some limited predictions about the subsequent fate of the plate: the four regimes range from waterexit to diving-entry into the fluid. In the examples presented for a thick steel plate, we show that the rigid-body components of the elastic-plate motion are very close to those of an equivalent rigid plate. That said, the elastic plate experiences higher forces owing to the higher velocity of its turnover region. The highest loads occur at the end of the impact, when the plate inclination approaches zero. The maximum bending stress exerted on the plate is found to be close to its yield stress.

An important conclusion in this study is that the flow and the motion of the plate should be solved simultaneously, in a model that couples them. If the problem is treated through a decoupled model, in which one applies to the elastic plate the pressure determined from the problem of rigid-plate impact, then the bending stresses are over-estimated. We found hydrodynamic pressures below atmospheric value in the contact region for the rigid-plate impact. It was shown that elastic vibrations of the plate lead to even lower pressures. It was confirmed that the shape of the free surface, the hydrodynamic pressure, and the flow, are all sensitive to such vibrations. We conclude from this that the elasticity of the plate promotes the inception of fluid cavitation - the detachment of the liquid boundary from the solid surface - during the early stage of impact. Cavitation is not included in the present analysis.

An account is made of the different energy components of the fluid-plate system as the impact proceeds. The work done to keep the horizontal velocity component of the plate constant is important and has to be accounted for in the energy balance. It is shown that a large part of the kinetic energy in the plate due to vertical motion is transferred into the jet. The potential energy of the plate due to bending is negligible compared to the kinetic energies associated with the mass of the plate and the fluid flow.

We finally discuss how the present work can be extended in at least three distinct ways: to include free-surface separation from near the trailing edge; a non-zero initial rotation rate of the plate; and realistic conditions of holding the plate in an experiment.

First, a free-surface separation point that moves near the trailing edge can influence the pressure distribution and the force on the plate. The position of such a free separation point must be determined as part of the solution. Equation (3.4) shows that the hydrodynamic pressure is proportional to the square-root of the distance from the trailing edge, with a coefficient which can be negative. The latter implies that the pressure in front of the trailing edge is below atmospheric pressure, which may lead to separation of the liquid surface from the solid surface of the plate.

Secondly, the initial angular speed of the plate is zero in the present paper, but it could be made non-zero: this may lead to different outcomes for the water-exit or diving-entry of the plate.

Finally, the plate can be considered as the bottom of a vehicle, or an experimental mounting, that is landing on water with high horizontal velocity. In such problems the boundary conditions at the ends of the plate can be modified to pinned or clamped conditions. Our modelling could also be extended to treat plates which are under longitudinal tension or which are more flexible than we have discussed in this paper. 


\section{Acknowledgements}

The authors thank Professor N. Riley for a discussion on the smallness of viscous drag, and we thank UEA Library for help accessing references. A.A.K. and M.J.C. were supported by Seventh Framework Programme (FP7), project number 266172. M.R. presented preliminary results at the 26th International Workshop on Water Waves and Floating Bodies, Athens, Greece, 2011, where he was supported by the Tuck Fellowship.

\section{Appendix. Numerical implementation}

The aim of this appendix is to present a numerical scheme for (3.3), (3.18), (3.31), (4.1), (4.4) and to indicate how to find the analytical forms of $\Lambda_{c}, \Gamma_{c}^{(1)}$ and $\Gamma_{c}^{(2)}$. Furthermore, we show a suitable evaluation of the hydrodynamic pressure acting on the plate.

\section{A.1. Numerical scheme}

Let us define $b_{k}(t)=\dot{a}_{k}(t)$ then (3.31) and (4.1) form an ordinary differential equation (ODE) system

$$
(\dot{\boldsymbol{a}}, \dot{\boldsymbol{b}}, \dot{\boldsymbol{c}})^{\mathrm{T}}=\boldsymbol{F}\left(t, \boldsymbol{a}, \boldsymbol{b}, c,\left.\bar{\varphi}_{x}(x)\right|_{x \in(0, t)},\left.A(x)\right|_{x \in(0, t)}\right),
$$

where $\bar{\varphi}_{x}(x)$ and $A(x)$ are assumed to be known in advance. We use the time discretization $t_{n}=n \Delta t$ with constant time step $\Delta t$ and $\boldsymbol{a}^{(n)}, \boldsymbol{b}^{(n)}, c^{(n)}$ are the numerical values of $\boldsymbol{a}\left(t_{n}\right), \boldsymbol{b}\left(t_{n}\right), c\left(t_{n}\right)$ for $n=0,1, \ldots, N$. We approximate $\bar{\varphi}_{x}(x)$ and $A(x)$ by constant values $\bar{\varphi}_{x}^{(n)}$ and $A^{(n)}$ in the intervals $x \in\left(t_{n-1}, t_{n}\right)$ for $n=1, \ldots, N$. The number of modes is limited in computations by setting $a_{k}=0$ for $k \geqslant M$. Only the first $M$ equations $k=0, \ldots, M-1$ in (3.31) are retained. In particular, we set $M=2$ to obtain results for the impact of a rigid plate. If the right-hand side of (3.30) is known, then this equation describes the forced oscillations of $a_{k}(t)$. The period of the eigen-oscillations is $T_{k}=2 \pi \lambda_{k}^{-2}(\mu / \theta)^{1 / 2}$. As $k$ increases the period $T_{k}=O\left(k^{-2}\right)$ decreases. Hence, $\Delta t$ has to be much smaller than $T_{M-1}$, which is the period of the highest mode retained in our computations. If the plate is light or very flexible $(\mu \ll 1$ or $\theta \ll 1)$ we expected the hydrodynamic pressure in (3.30) to strongly depend on the plate deflection, and the problem is then strongly coupled.

For each time step the numerical solver consists of two stages. In the first stage we use a modified Euler's method to integrate (A 1):

$$
\begin{gathered}
\boldsymbol{F}_{0}^{(n-1)}=\boldsymbol{F}\left(t_{n-1}, \boldsymbol{a}^{(n-1)}, \boldsymbol{b}^{(n-1)}, c^{(n-1)} ; \bar{\varphi}_{x}^{(1)}, \ldots, \bar{\varphi}_{x}^{(n-1)} ; A^{(1)}, \ldots, A^{(n-1)}\right), \\
\left(\tilde{\boldsymbol{a}}^{(n)}, \tilde{\boldsymbol{b}}^{(n)}, \tilde{c}^{(n)}\right)=\left(\boldsymbol{a}^{(n-1)}, \boldsymbol{b}^{(n-1)}, c^{(n-1)}\right)+\Delta t \boldsymbol{F}_{0}^{(n-1)}, \\
\boldsymbol{F}_{1}^{(n-1)}=\boldsymbol{F}\left(t_{n}, \tilde{\boldsymbol{a}}^{(n)}, \tilde{\boldsymbol{b}}^{(n)}, \tilde{\boldsymbol{c}}^{(n)} ; \bar{\varphi}_{x}^{(1)}, \ldots, \bar{\varphi}_{x}^{(n-1)}, \bar{\varphi}_{x}^{(n-1)} ; A^{(1)}, \ldots, A^{(n-1)}, A^{(n-1)}\right), \\
\left(\boldsymbol{a}^{(n)}, \boldsymbol{b}^{(n)}, c^{(n)}\right)=\left(\boldsymbol{a}^{(n-1)}, \boldsymbol{b}^{(n-1)}, c^{(n-1)}\right)+\frac{\Delta t}{2}\left(\boldsymbol{F}_{0}^{(n-1)}+\boldsymbol{F}_{1}^{(n-1)}\right) .
\end{gathered}
$$

Note that we use $\bar{\varphi}_{x}^{(n-1)}$ and $A^{(n-1)}$ as the $n$th input for $\bar{\varphi}_{x}(x)$ and $A(x)$ in (A 4), so that an error in $\boldsymbol{F}_{1}^{(n-1)}$ of order $(\Delta t)^{5 / 2}$ is introduced (see (3.31) and (4.1)). In the second stage we solve the discretized integral equations (3.3) and (3.18) by

$$
\bar{\varphi}_{x}^{(n)}=\frac{1}{\beta_{n n}^{(1)}}\left(\sum_{k=0}^{M} b_{k}^{(n)} \Gamma_{c_{n}}^{(2)}\left(\psi_{k}\right)-\sum_{m=1}^{n-1} \beta_{m n}^{(1)} \bar{\varphi}_{x}^{(m)}\right),
$$




$$
A^{(n)}=\frac{1}{\beta_{n n}^{(1)}}\left(\sum_{k=0}^{M} a_{k}^{(n)} \Gamma_{c_{n}}^{(2)}\left(\psi_{k}\right)-\sum_{m=1}^{n-1} \beta_{m n}^{(1)} A^{(m)}-\sum_{m=1}^{n} \beta_{m n}^{(2)} \bar{\varphi}_{x}^{(m)}\right)
$$

to obtain $\bar{\varphi}_{x}^{(n)}$ and $A^{(n)}$ where

$$
\beta_{m n}^{(1)}=\int_{t_{m-1}}^{t_{m}} \sqrt{\frac{t_{n}+c_{n}-\xi}{t_{n}-\xi}} \mathrm{d} \xi, \quad \beta_{m n}^{(2)}=\int_{t_{m-1}}^{t_{m}} \sqrt{\left(t_{n}+c_{n}-\xi\right)\left(t_{n}-\xi\right)} \mathrm{d} \xi .
$$

A discussion of convergence of the product-integration method for Volterra integral equations of the first kind presented in (A 6) and (A 7) can be found in Baker (1977).

Equations (A 2)-(A 7) are used starting from $n=2$. At $t=\Delta t, n=1$, the unknown values are approximated by those for the oblique impact of a rigid plate with constant velocity (see (5.1) and (5.2)). We confirmed numerically that the time-derivatives of the principal coordinates, $\dot{a}_{k}$, converge as $O\left((\Delta t)^{2}\right)$ and $\bar{\varphi}_{x}$ converges as $O(\Delta t)$ as $\Delta t \rightarrow 0$.

\section{A.2. Evaluation of $\Lambda_{c}, \Gamma_{c}^{(1)}$ and $\Gamma_{c}^{(2)}$}

In (3.32)-(3.35), (4.1) and (4.3) the terms $\Lambda_{c}\left(\psi_{k}, \psi_{l}\right), \Gamma_{c}^{(1)}\left(\psi_{k}\right)$ and $\Gamma_{c}^{(2)}\left(\psi_{k}\right)$ for $k, l \geqslant 2$ can be expressed as linear combinations of the following identities:

$$
\begin{gathered}
\Lambda_{c}\left(\mathrm{e}^{r_{1} x}, \mathrm{e}^{r_{2} x}\right)=\frac{\pi c r_{1}}{2\left(r_{1}+r_{2}\right)}\left(\mathrm{I}_{0}\left(\frac{c r_{2}}{2}\right) \mathrm{I}_{1}\left(\frac{c r_{1}}{2}\right)+\mathrm{I}_{0}\left(\frac{c r_{1}}{2}\right) \mathrm{I}_{1}\left(\frac{c r_{2}}{2}\right)\right) \mathrm{e}^{c\left(r_{1}+r_{2}\right) / 2}, \\
\Gamma_{c}^{(1)}\left(\mathrm{e}^{r x}\right)=\frac{\pi c}{2}\left(\mathrm{I}_{0}\left(\frac{c r}{2}\right)+\mathrm{I}_{1}\left(\frac{c r}{2}\right)\right) \mathrm{e}^{c r / 2}, \\
\Gamma_{c}^{(2)}\left(\mathrm{e}^{r x}\right)=\frac{\pi c}{2}\left(\mathrm{I}_{0}\left(\frac{c r}{2}\right)-\mathrm{I}_{1}\left(\frac{c r}{2}\right)\right) \mathrm{e}^{c r / 2},
\end{gathered}
$$

where $r, r_{1}, r_{2}$ are complex constants, and $\mathrm{I}_{j}(x)$ are modified Bessel functions of the first kind. For $k=0,1$ or $l=0,1$ the integrals $\Lambda_{c}\left(\psi_{k}, \psi_{l}\right), \Gamma_{c}^{(1)}\left(\psi_{k}\right)$ and $\Gamma_{c}^{(2)}\left(\psi_{k}\right)$ are much simpler but we do not go into detail here.

\section{A.3. Evaluation of the vertical hydrodynamic force, moment and pressure}

Equation (3.30) for $k=0$ and $k=1$ provides the following formulae for the hydrodynamic force $F(t)=\int_{0}^{c} p(s+t, 0, t) \mathrm{d} s$ acting on the plate and the moment $M(t)=\int_{0}^{c} s p(s+t, 0, t) \mathrm{d} s$ about the trailing edge in terms of the principal coordinates of the rigid modes $a_{0}$ and $a_{1}$ :

$$
F(t)=\mu\left(\ddot{a}_{0}(t)+\kappa\right), \quad M(t)=\frac{\mu}{2}\left(\frac{1}{\sqrt{3}} \ddot{a}_{1}(t)+\ddot{a}_{0}(t)+\kappa\right) .
$$

We find the pressure by evaluating $B(t)$ and $T(s+t, t)$ and applying (3.4). The function $B(t)$ is given by (4.3). The function $T(s+t, t)$ can be written as

$$
T(s+t, t)=\left(\boldsymbol{a}^{\mathrm{T}} \boldsymbol{P}(c)-2 \dot{\boldsymbol{a}}^{\mathrm{T}} \boldsymbol{Q}(c)+\ddot{\boldsymbol{a}}^{\mathrm{T}} \boldsymbol{R}(c)\right) \boldsymbol{U}(2 s / c-1),
$$

where $0<s<c$ and the elements of the vector $\boldsymbol{U}(x)=\left(U_{0}(x), U_{1}(x), \ldots\right)^{\mathrm{T}}$ are the Chebyshev polynomials of the second kind. The elements of the matrices $\boldsymbol{P}, \boldsymbol{Q}$ and $\boldsymbol{R}$ are defined as the coefficients in the following series:

$$
f_{0}^{c} \frac{\psi_{k}^{\prime}(u)}{(u-s) \sqrt{u(c-u)}} \mathrm{d} u=\sum_{j=0}^{\infty} P_{k j}(c) U_{j}(2 s / c-1),
$$




$$
\begin{aligned}
& f_{0}^{c} \frac{\psi_{k}(u)}{(u-s) \sqrt{u(c-u)}} \mathrm{d} u=\sum_{j=0}^{\infty} Q_{k j}(c) U_{j}(2 s / c-1), \\
& f_{0}^{c} \frac{\Psi_{k}(u)}{(u-s) \sqrt{u(c-u)}} \mathrm{d} u=\sum_{j=0}^{\infty} R_{k j}(c) U_{j}(2 s / c-1) .
\end{aligned}
$$

Note that these functions depend on the size of the contact region but not on the impact conditions. This implies that these functions can be pre-computed for $0 \leqslant c \leqslant 1$. For $k=0,1$ the left-hand sides of (A 14), (A 15) and (A 16) are linear polynomials in $s$ and can be expressed by a linear combination of $U_{0}$ and $U_{1}$, so that $P_{k j}=Q_{k j}=R_{k j}=0$ for $j \geqslant 2$. To determine the elements $P_{k j}, Q_{k j}$ and $R_{k j}$ for $k \geqslant 2$, we derived the identity

$$
f_{0}^{c} \frac{\mathrm{e}^{r u}}{(u-s) \sqrt{u(c-u)}} \mathrm{d} u=\frac{4 \pi}{c} \mathrm{e}^{r c / 2} \sum_{j=0}^{\infty} \mathrm{I}_{j+1}\left(\frac{r c}{2}\right) U_{j}(2 s / c-1) .
$$

For example, with the help of expression (3.27) for the normal modes $\psi_{k}(s)$ together with formula (A 17) for $r= \pm \lambda_{k}, \pm \mathrm{i} \lambda_{k}$ we obtain

$$
\begin{aligned}
& Q_{k j}(c)= \frac{4 \pi}{c}\left(\mathrm{e}^{-\lambda_{k} c / 2} \mathrm{I}_{j+1}\left(\frac{\lambda_{k} c}{2}\right) \frac{\mathrm{e}^{-\lambda_{k}(1-c)}+(-1)^{k+j+1}}{(-1)^{k}+\mathrm{e}^{-\lambda_{k}}}+f_{k} \mathrm{~J}_{j+1}\left(\frac{\lambda_{k} c}{2}\right)\right), \\
& f_{k}=\left\{\begin{array}{lc}
\cos \left[\frac{\lambda_{k}}{2}(1-c)-\frac{\pi}{2}(j+1)\right] / \cos \left(\frac{\lambda_{k}}{2}\right) & \text { for } k \text { even, } \\
\sin \left[\frac{\lambda_{k}}{2}(1-c)-\frac{\pi}{2}(j+1)\right] / \sin \left(\frac{\lambda_{k}}{2}\right) & \text { for } k \text { odd, }
\end{array}\right.
\end{aligned}
$$

for $k \geqslant 2$. The functions $\mathrm{J}_{j}(x)$ are Bessel functions of the first kind. Similar formulae were derived for $P_{k j}(c)$ and $R_{k j}(c)$. For large $k$ and $j$ in (A 18) the functions $\mathrm{J}_{j}(x)$ and $\mathrm{e}^{-x} \mathrm{I}_{j}(x)$ are bounded for $x>0$ and tend uniformly to zero as $j \rightarrow \infty$. For lower $k$ the convergence of $Q_{k j}$ towards zero, as $j$ increases, is even stronger. In general, we obtained good results when we set $P_{k j}=Q_{k j}=R_{k j}=0$ for $j \geqslant 10$. For large $k$ the values $P_{k j} a_{k}, Q_{k j} \dot{a}_{k}$ and $R_{k j} \ddot{a}_{k}$ in (A 13) give negligible contributions.

\section{REFERENCES}

ACHeson, D. J. 1990 Elementary Fluid Dynamics. Clarendon.

BAKER, C. T. 1977 The Numerical Treatment of Integral Equations. Clarendon.

Birkhoff, G. \& Zarantonello, E. H. 1957 Jets, Wakes, and Cavities. Academic.

Cointe, R., Fontaine, E., Molin, B. \& Scolan, Y.-M. 2004 On energy arguments applied to the hydrodynamic impact force. J. Engng Maths 48, 305-319.

COOKER, M. J. 1996 Sudden changes in a potential flow with a free surface due to impact. Q. J. Mech. Appl. Maths 49 (4), 581-591.

Donnell, L. H. 1976 Beams, Plates and Shells. McGraw-Hill.

FAltinsen, O. M. 2005 Hydrodynamics of High-Speed Marine Vehicles. Cambridge University Press.

Faltinsen, O. M., KVÅlsvold, J. \& Aarsnes, J. 1997 Wave impact on a horizontal elastic plate. J. Mar. Sci. Technol. 2, 87-100.

FAltinsen, O. M. \& Semenov, Y. A. 2008 Nonlinear problem of flat-plate entry into an incompressible liquid. J. Fluid Mech. 611, 151-173.

GAKhov, F. D. 1966 Boundary Value Problems. Pergamon.

Hewitt, I. J., Balmforth, N. J. \& McElwaine, J. N. 2011 Continual skipping on water. J. Fluid Mech. 669, 328-353. 
Hicks, P. D. \& PURVis, R. 2010 Air cushioning and bubble entrapment in three-dimensional droplet impacts. J. Fluid Mech. 649, 135-163.

Hicks, P. D. \& Sмith, F. T. 2011 Skimming impacts and rebounds on shallow liquid layers. Proc. R. Soc.Lond. A 467, 653-674.

Howison, S. D., Morgan, J. D. \& OCkendon, J. R. 1997 A class of codimension-two free boundary problems. SIAM Rev. 39 (2), 221-253.

Howison, S. D., Ockendon, J. R. \& Oliver, J. M. 2004 Oblique slamming, planing and skimming. J. Engng Maths 48, 321-337.

Howison, S. D., OCKEndon, J. R. \& Wilson, S. K. 1991 Incompressible water-entry problems at small deadrise angles. J. Fluid Mech. 222, 215-230.

IAfrati, A. \& Korobin, A. A. 2008 Hydrodynamic loads during early stage of flat plate impact onto water surface. Phys. Fluids 20, 082104.

Khabakhpasheva, T. I. \& Korobin, A. A. 2013 Oblique impact of a smooth body on a thin layer of inviscid liquid. Proc. R. Soc.Lond. A 469 (2151).

KInG, F. W. 2009 Hilbert Transforms, Vol. 1. Cambridge University Press.

Korob Kin, A. A. 1994 Blunt-body penetration into a slightly compressible liquid. In Proceedings of 20th Symposium on Naval Hydrodynamics, Santa Barbara, pp. 179-186. Office of Naval Research.

Korob Kin, A. A. 1995 Wave impact on the bow end of a catamaran wet deck. J. Ship Res. 39 (4), 321-327.

Korob Kin, A. A. 1998 Wave impact on the centre of an Euler beam. J. Appl. Mech. Tech. Phys. 39, 770-781.

Korobkin, A. A. 2004 Analytical models of water impact. Eur. J. Appl. Maths 15, 821-838.

Korobinin, A. A. 2007 Second-order Wagner theory of wave impact. J. Engng Maths 58, 121-139.

Korobin, A. A. \& Khabakhpasheva, T. I. 2006 Regular wave impact onto an elastic plate. J. Engng Maths 55, 127-150.

Meyerhoff, W. K. $1965 a$ Die Berechnung hydroelastischer Stöße. Schiffstechnik 12 60, 18-30.

Meyerhoff, W. K. $1965 b$ Die Berechnung hydroelastischer Stöße. Schiffstechnik 12 61, 49-64.

Moore, M. R., Howison, S. D., Ockendon, J. R. \& Oliver, J. M. $2012 a$ A note on oblique water entry. J. Engng Maths doi:10.1007/s10665-012-9570-0.

Moore, M. R., Howison, S. D., Ockendon, J. R. \& Oliver, J. M. $2012 b$ Three-dimensional oblique water-entry problems at small deadrise angles. J. Fluid Mech. 711, 259-280.

NeWman, J. N. 1977 Marine Hydrodynamics. MIT.

OLIVER, J. M. 2002 Water entry and related problems. D.Phil thesis, University of Oxford.

OLIVER, J. M. 2007 Second-order Wagner theory for two-dimensional water-entry problems at small deadrise angles. J. Fluid Mech. 572, 59-85.

Reinhard, M., Korobkin, A. \& Cooker, M. J. $2012 a$ The bounce of a blunt body from a water surface at high horizontal speed. In 27th International Workshop on Water Waves and Floating Bodies, Copenhagen, pp. 153-156. Technical University of Denmark.

Reinhard, M., Korobkin, A. A. \& Cooker, M. J. $2012 b$ Elastic plate impact into water at high horizontal speed with early water detachment. In 6th International Conference on Hydroelasticity in Marine Technology 2012, Tokyo, pp. 1-10. University of Tokyo Press.

Rosselini, L., Hersen, F., Clanet, C. \& Bocquet, L. 2005 Skipping stones. J. Fluid Mech. 543, $137-146$.

Scolan, Y.-M. \& KorobKin, A. A. 2003 Energy distribution from vertical impact of a three-dimensional solid body onto the flat free surface of an ideal fluid. J. Fluids Struct. 17 (2), 275-286.

ScOlan, Y.-M. \& Korob Kin, A. A. 2012 Hydrodynamic impact (Wagner) problem and Galin's theorem. In 27th International Workshop on Water Waves and Floating Bodies, Copenhagen. Technical University of Denmark.

Sedov, L. I. 1940 On the theory of unsteady planing and the motion of a wing with vortex separation. NACA Tech Rep. 942.

Semenov, Y. A. \& Yoon, B. S. 2009 Onset of flow separation for the oblique water impact of a wedge. Phys. Fluids 21, 112103. 
SMILEY, R. F. 1951 An experimental study of water-pressure distributions during landings and planing of a heavily loaded rectangular flat-plate model. NACA Tech Rep. 2453.

ULSTEIN, T. 1995 Nonlinear effects of a flexible stern seal bag on cobblestone oscillations of an SES. PhD thesis, Norwegian Institute of Technology, Trondheim.

Ulstein, T. \& Faltinsen, O. M. 1996 Two-dimensional unsteady planing. J. Ship Res. 40 (3), 200-210.

Vorus, W. S. 1996 A flat cylinder theory for vessel impact and steady planing resistance. J. Ship Res. 40 (2), 89-106.

WAGNER, H. 1932 Über Stoß- und Gleitvorgänge an der Oberfläche von Flüssigkeiten. Z. Angew. Math. Mech. 12, 193-215.

Zhao, R. \& FAltinsen, O. M. 1993 Water entry of two-dimensional bodies. J. Fluid Mech. 246, 593-612.

ZhaO, R., FAltinsen, O. M. \& AARSNES, J. 1996 Water entry of arbitrary two-dimensional sections with and without flow separation. In Proceedings of 21 st Symposium on Naval Hydrodynamics, Trondheim, pp. 408-423, US National Research Council. 